\title{
HADAMARD COMPOSITIONS OF GELFOND-LEONT'EV-SǍLǍGEAN AND GELFOND-LEONT'EV-RUSCHEWEYH DERIVATIVES OF FUNCTIONS ANALYTIC IN THE UNIT DISK
}

M. M. Sheremeta. Hadamard compositions of Gelfond-Leont'ev-Sălăgean and Gelfond-Leont'ev-Ruscheweyh derivatives of functions analytic in the unit disk, Mat. Stud. 54 (2020), 115134.

For analytic functions

$$
f(z)=z+\sum_{k=2}^{\infty} f_{k} z^{k} \text { and } g(z)=z+\sum_{k=2}^{\infty} g_{k} z^{k}
$$

in the unit disk properties of the Hadamard compositions $D_{l,[S]}^{n} f * D_{l,[S]}^{n} g$ and $D_{l,[R]}^{n} f * D_{l,[R]}^{n} g$ of their Gelfond-Leont'ev-Sălăgean derivatives

$$
D_{l,[S]}^{n} f(z)=z+\sum_{k=2}^{\infty}\left(\frac{l_{1} l_{k-1}}{l_{k}}\right)^{n} f_{k} z^{k}
$$

and Gelfond-Leont'ev-Ruscheweyh derivatives

$$
D_{l,[R]}^{n} f(z)=z+\sum_{k=2}^{\infty} \frac{l_{k-1} l_{n}}{l_{n+k-1}} f_{k} z^{k}
$$

are investigated. For study, generalized orders are used. A connection between the growth of the maximal term of the Hadamard composition of Gelfond-Leont'ev-Sălăgean derivatives or Gelfond-Leont'ev-Ruscheweyh derivatives and the growth of the maximal term of these derivatives of Hadamard composition is established. Similar results are obtained in terms of the classical order and the lower order of the growth.

1. Introduction. For formal power series

$$
f(z)=\sum_{k=0}^{\infty} f_{k} z^{k} \text { and } l(z)=\sum_{k=0}^{\infty} l_{k} z^{k}
$$

$\left(l_{k}>0\right)$ the formal power series

$$
D_{l}^{n} f(z)=\sum_{k=0}^{\infty} \frac{l_{k}}{l_{k+n}} f_{k+n} z^{k}
$$

2010 Mathematics Subject Classification: 30B10, 30B40.

Keywords: analytic function; Gelfond-Leont'ev-Sălăgean derivative; Gelfond-Leont'ev-Ruscheweyh derivative; Hadamard composition; generalized order.

doi:10.30970/ms.54.2.115-134

C) M. M. Sheremeta, 2020 
is called the Gelfond-Leont'ev derivative ([1]). If $l(z)=e^{z}$ (i.e. $l_{k}=1 / k !$ ) then $D_{l}^{n} f=f^{(n)}$ is a usual derivative.

Let $H$ be the class of analytic in the disk $\{z:|z|<1\}$ functions given by power series

$$
f(z)=z+\sum_{k=2}^{\infty} f_{k} z^{k}
$$

with the radius of convergence $R[f]=1$ and the operator $D_{[S]}^{n} f(n \geq 0)$ be defined by $D_{[S]}^{0} f(z)=f(z), D_{[S]}^{1} f(z)=D_{[S]} f(z)=z f^{\prime}(z)$ and

$$
D_{[S]}^{n} f(z)=D_{[S]}\left(D_{[S]}^{n-1} f(z)\right)=z+\sum_{k=2}^{\infty} k^{n} f_{k} z^{k} .
$$

The operator $D_{[S]}^{n} f$ is known as the Sălăgean derivative ([2]). For $f \in H$

$$
D_{[R]}^{n} f(z)=\frac{z}{n !} \frac{d^{n}}{d z^{n}}\left\{z^{n-1} f(z)\right\}=z+\sum_{k=2}^{\infty} \frac{(k+n-1) !}{n !(k-1) !} f_{k} z^{k}
$$

is called the Ruscheweyh derivative ([3]).

Combining the definitions of Gelfond-Leont'ev derivative with Sălăgean derivative and Ruscheweyh derivative in [4] for $f \in H$ the following operators are defined

$$
D_{l,[S]}^{n} f(z)=l_{1} z D_{l}^{1}\left(D_{l,[S]}^{n-1} f(z)\right)=z+\sum_{k=2}^{\infty}\left(\frac{l_{1} l_{k-1}}{l_{k}}\right)^{n} f_{k} z^{k}
$$

and

$$
D_{l,[R]}^{n} f(z)=z l_{n} D_{l}^{n}\left\{z^{n-1} f(z)\right\}=z+\sum_{k=2}^{\infty} \frac{l_{k-1} l_{n}}{l_{n+k-1}} f_{k} z^{k} .
$$

The operator $D_{l,[S]}^{n}$ is called the Gelfond-Leont'ev-Sălăgean derivative ([4]) and the operator $D_{l,[R]}^{n}$ is called the Gelfond-Leont'ev-Ruscheweyh derivative. series

For power series (1) and $g(z)=\sum_{k=0}^{\infty} g_{k} z^{k}$ with the convergence radii $R[f]$ and $R[g]$ the

$$
(f * g)(z)=\sum_{k=0}^{\infty} f_{k} g_{k} z^{k}
$$

is called [5, 6] the Hadamard composition. Obtained by J. Hadamard properties of this composition find the applications $([6,7])$ in the theory of the analytic continuation of the functions represented by power series. We remark also that singular points of the Hadamard composition are investigated in the paper [8].

For $0 \leq r<R[f]$ let $M(r, f)=\max \{|f(z)|:|z|=r\}$ and $\mu(r, f)=\max \left\{\left|f_{k}\right| r^{k}: k \geq 0\right\}$ be the maximal term of the power expansion of $f$ and $\nu(r, f)=\max \left\{n: \mid f_{n} r^{n}=\mu(r, f)\right\}$ be its central index. A connection between the growth of the maximal terms of a derivative of the Hadamard composition of two entire functions $f$ and $g$ and the Hadamard composition of their derivative is studied by M. K. Sen $([9,10])$. The properties of compositions of Hadamard for Gelfond-Leont'ev derivatives of analytic functions $f$ and $g$ are investigated in [11]. For entire functions $f$ and $g$ it is proved ([11]) for example that if

$$
0<\varliminf_{k \rightarrow \infty} \frac{l_{k}}{(k+1) l_{k+1}} \leq \varlimsup_{k \rightarrow \infty} \frac{l_{k}}{(k+1) l_{k+1}}<+\infty
$$


then

$$
\varlimsup_{r \rightarrow+\infty} \frac{1}{\ln r} \ln \frac{\mu\left(r, D_{l}^{(n+1)} f * D_{l}^{(n+1)} g\right)}{\mu\left(r, D_{l}^{(n)}(f * g)\right)}=(n+2) \varrho[f * g]-1
$$

and

$$
\varliminf_{r \rightarrow+\infty} \frac{1}{\ln r} \ln \frac{\mu\left(r, D_{l}^{(n+1)} f * D_{l}^{(n+1)} g\right)}{\mu\left(r, D_{l}^{(n)}(f * g)\right)}=(n+2) \lambda[f * g]-1,
$$

where $\varrho[f]$ is the order and $\lambda[f]$ is the lower order of the entire function $f$. If $R[f]=1$, $R[g]=1$ and $R[f * g]=1$ then $([11])$

$$
(n+2) \varrho^{(1)}[f * g] \leq \varlimsup_{r \uparrow 1} \frac{1}{-\ln (1-r)} \ln ^{+} \frac{\mu\left(r, D_{l}^{(n+1)} f * D_{l}^{(n+1)} g\right)}{\mu\left(r, D_{l}^{(n)}(f * g)\right)} \leq(n+2)\left(\varrho^{(1)}[f * g]+1\right)
$$

and

$$
(n+2) \lambda^{(1)}[f * g] \leq \frac{\lim }{r \uparrow 1} \frac{1}{-\ln (1-r)} \ln ^{+} \frac{\mu\left(r, D_{l}^{(n+1)} f * D_{l}^{(n+1)} g\right)}{\mu\left(r, D_{l}^{(n)}(f * g)\right)} \leq(n+2)\left(\lambda^{(1)}[f * g]+1\right),
$$

where $\varrho^{(1)}[f]$ is the order and $\lambda^{(1)}[f]$ is the lower order of the analytic function $f$ in the unit disk.

Naturally, the question arises of similar properties of the Hadamard compositions of the Gelfond-Leont'ev-Sălăgean derivatives and the Gelfond-Leont'ev-Ruscheweyh derivatives.

2. Hadamard composition of two Gelfond-Leont'ev-Sălăgean derivatives. Let $f \in$ $H, g \in H, n \in \mathbb{Z}_{+}$and $m \in \mathbb{Z}_{+}$. Then

$$
\begin{gathered}
\left(D_{l,[S]}^{n} f * D_{l,[S]}^{m} g\right)(z)=z+\sum_{k=2}^{\infty}\left(\frac{l_{1} l_{k-1}}{l_{k}}\right)^{n} f_{k}\left(\frac{l_{1} l_{k-1}}{l_{k}}\right)^{m} g_{k} z^{k}= \\
=z+\sum_{k=2}^{\infty}\left(\frac{l_{1} l_{k-1}}{l_{k}}\right)^{n+m} f_{k} g_{k} z^{k}=D_{l,[S]}^{n+m}(f * g)(z),
\end{gathered}
$$

i. e. the study of the Hadamard composition of Gelfond-Leont'ev-Sălăgean derivatives of two functions is reduced to the study of Gelfond-Leont'ev-Sălăgeanthe derivative of the Hadamard composition of these functions. For the Gelfond-Leont'ev-Sălăgeanthe derivative of the Hadamard composition the following statement is true.

Lemma 1. If $f \in H, g \in H, n \in \mathbb{N}$ and there exists $\lim _{k \rightarrow \infty} \sqrt[k]{l_{k-1} / l_{k}}=q$ then $R\left[D_{l,[S]}^{n}(f * g)\right] \geq$ $q^{-n}$ and if, moreover, $\lim _{k \rightarrow \infty} \sqrt[k]{\left|g_{k}\right|}=1$ then $R\left[D_{l,[S]}^{n}(f * g)\right]=q^{-n}$.

Proof. Indeed,

$$
\begin{gathered}
\frac{1}{R\left[D_{l,[S]}^{n}(f * g)\right]}=\varlimsup_{k \rightarrow \infty} \sqrt[k]{\left(\frac{l_{1} l_{k-1}}{l_{k}}\right)^{n}\left|f_{k}\right|\left|g_{k}\right|} \leq \\
\leq\left(\varlimsup_{k \rightarrow \infty} \sqrt[k]{\frac{l_{k-1}}{l_{k}}}\right)^{n} \varlimsup_{k \rightarrow \infty} \sqrt[k]{\left|f_{k}\right|} \varlimsup_{k \rightarrow \infty} \sqrt[k]{\left|g_{k}\right|}=\left(\varlimsup_{k \rightarrow \infty} \sqrt[k]{\frac{l_{k-1}}{l_{k}}}\right)^{n}, \\
\frac{1}{R\left[D_{l,[S]}^{n}(f * g)\right]} \geq\left(\varliminf_{k \rightarrow \infty} \sqrt[k]{\frac{l_{k-1}}{l_{k}}}\right)^{n} \varlimsup_{k \rightarrow \infty} \sqrt[k]{\left|f_{k}\right|} \underline{\lim _{k \rightarrow \infty}} \sqrt[k]{\left|g_{k}\right|} .
\end{gathered}
$$


Therefore, if there exists $\lim _{k \rightarrow \infty} \sqrt[k]{l_{k-1} / l_{k}}=q$ then $1 / R\left[D_{l,[S]}^{n}(f * g)\right] \leq q^{n}$, and if, moreover, $\lim _{k \rightarrow \infty} \sqrt[k]{\left|g_{k}\right|}=1$ then $1 / R\left[D_{l,[S]}^{n}(f * g)\right] \geq q^{n}$. Lemma 1 is proved.

Let $E$ be the class of entire functions. From Lemma 1 it follows that if $\sqrt[k]{l_{k} / l_{k-1}} \rightarrow \infty$ as $k \rightarrow \infty$ then $D_{l,[S]}^{n}(f * g) \in E$. If $\sqrt[k]{l_{k} / l_{k-1}} \rightarrow 1$ and $\lim _{k \rightarrow \infty} \sqrt[k]{\left|g_{k}\right|}=1$ as $k \rightarrow \infty$ then $D_{l,[S]}^{n}(f * g) \in H$. In the sequel, we will consider only these two cases.

To study the growth of analytic functions, we will use generalized orders. For this purpose by $L$ we denote the class of continuous non-negative on $(-\infty,+\infty)$ functions $\alpha$ such that $\alpha(x)=\alpha\left(x_{0}\right)>0$ for $x \leq x_{0}$ and $\alpha(x) \uparrow+\infty$ as $x_{0} \leq x \rightarrow+\infty$. We say that $\alpha \in L^{0}$, if $\alpha \in L$ and $\alpha((1+o(1)) x)=(1+o(1)) \alpha(x)$ as $x \rightarrow+\infty$. Finally, $\alpha \in L_{s i}$, if $\alpha \in L$ and $\alpha(c x)=(1+o(1)) \alpha(x)$ as $x \rightarrow+\infty$ for each fixed $c \in(0,+\infty)$, i. e. $\alpha$ is a slowly increasing function. Clearly, $L_{s i} \subset L^{0}$.

2.1. The case $\lim _{\mathbf{k} \rightarrow \infty} \sqrt[k]{l_{k} / l_{k-1}}=+\infty$. For $\alpha \in L, \beta \in L$ and an entire transcendental function (1) the quantities

$$
\begin{aligned}
& \varrho_{\alpha, \beta}[f]:=\varrho_{\alpha, \beta}[\ln M, f]=\varlimsup_{r \rightarrow+\infty} \frac{\alpha(\ln M(r, f))}{\beta(\ln r)}, \\
& \lambda_{\alpha, \beta}[f]:=\lambda_{\alpha, \beta}[\ln M, f]=\varliminf_{r \rightarrow+\infty} \frac{\alpha(\ln M(r, f))}{\beta(\ln r)}
\end{aligned}
$$

are called ([12]) the generalized order and the lower generalized order, respectively. If we substitute $\ln \mu(r, f)$ or $\nu(r, f)$ instead of $\ln M(r, f)$ then we obtain the definitions of the quantities $\varrho_{\alpha, \beta}[\ln \mu, f], \lambda_{\alpha, \beta}[\ln \mu, f]$ and $\lambda_{\alpha, \beta}[\nu, f], \lambda_{\alpha, \beta}[\nu, f]$, respectively.

Lemma 2. Let $\alpha \in L_{s i}, \beta \in L^{0}$ and $\frac{d \beta^{-1}(c \alpha(x))}{d \ln x}=O(1)$ as $x \rightarrow+\infty$ for each $c \in(0,+\infty)$. Then

$$
\varrho_{\alpha, \beta}[f]=\varlimsup_{k \rightarrow \infty} \alpha(k) / \beta\left(\frac{1}{k} \ln \frac{1}{\left|f_{k}\right|}\right) .
$$

If, moreover, $\left|f_{k} / f_{k+1}\right| \nearrow+\infty$ as $k_{0} \leq k \rightarrow \infty$ then

$$
\lambda_{\alpha, \beta}[f]=\varliminf_{k \rightarrow \infty} \alpha(k) / \beta\left(\frac{1}{k} \ln \frac{1}{\left|f_{k}\right|}\right) .
$$

Formula (5) is proved in [12], and formula (6) follows from the corresponding formula for entire Dirichlet series proved in [13].

The following lemma is proved in [14].

Lemma 3. If $\alpha \in L_{s i}$ and $\beta \in L^{0}$ then $\varrho_{\alpha, \beta}[f]=\varrho_{\alpha, \beta}[\ln \mu, f]$ and $\lambda_{\alpha, \beta}[f]=\lambda_{\alpha, \beta}[\ln \mu, f]$. If, moreover, $\alpha\left(e^{x}\right) \in L_{s i}$ and $\alpha(x)=o(\beta(x))$ as $x \rightarrow+\infty$ then $\varrho_{\alpha, \beta}[\ln \mu, f]=\varrho_{\alpha, \beta}[\nu, f]$ and $\lambda_{\alpha, \beta}[\ln \mu, f]=\lambda_{\alpha, \beta}[\nu, f]$.

Theorem 1. Let $\alpha(x)=\alpha_{1}(\ln x), \alpha_{1} \in L_{s i}, \beta \in L_{s i}$ and $\frac{d \beta^{-1}(c \alpha(x))}{d \ln x}=O(1)$ as $x \rightarrow+\infty$ for each $c \in(0,+\infty)$. Suppose that $\varliminf_{k \rightarrow \infty} \sqrt[k]{\left|f_{k} g_{k}\right|}>0$ and

$$
0<q=\varliminf_{k \rightarrow \infty} \frac{1}{\ln k} \ln \ln \frac{l_{k}}{l_{k-1}} \leq \varlimsup_{k \rightarrow \infty} \frac{1}{\ln k} \ln \ln \frac{l_{k}}{l_{k-1}}=Q<+\infty .
$$


Then for $m>n \geq 1$

$$
\varlimsup_{r \rightarrow+\infty} \frac{1}{\beta(\ln r)} \alpha\left(\ln \frac{\mu\left(r, D_{l,[S]}^{n}(f * g)\right)}{\mu\left(r, D_{l,[S]}^{m}(f * g)\right)}\right)=\varlimsup_{k \rightarrow \infty} \frac{\alpha(k)}{\beta\left(\frac{1}{k} \ln \frac{l_{k}}{l_{k-1}}\right)}
$$

and if, moreover, $\left|f_{k} / f_{k+1}\right| \nearrow 1,\left|g_{k} / g_{k+1}\right| \nearrow 1$ and $l_{k-1} l_{k+1} / l_{k}^{2} \nearrow+\infty$ as $k_{0} \leq k \rightarrow \infty$ then

$$
\varliminf_{r \rightarrow+\infty} \frac{1}{\beta(\ln r)} \alpha\left(\ln \frac{\mu\left(r, D_{l,[S]}^{n}(f * g)\right)}{\mu\left(r, D_{l,[S]}^{m}(f * g)\right)}\right)=\varliminf_{k \rightarrow \infty} \frac{\alpha(k)}{\beta\left(\frac{1}{k} \ln \frac{l_{k}}{l_{k-1}}\right)} .
$$

Proof. Using the definitions of the maximal term and the central index, we have

$$
\begin{gathered}
\mu\left(r, D_{l,[S]}^{n}(f * g)\right)=\left(\frac{l_{1} l_{\nu\left(r, D_{l,[S]}^{n}(f * g)\right)-1}}{l_{\nu\left(r, D_{l,[S]}^{n}(f * g)\right)}}\right)^{n}\left|f_{\nu\left(r, D_{l,[S]}^{n}(f * g)\right.}\right|\left|g_{\nu\left(r, D_{l,[S]}^{n}(f * g)\right)}\right| r^{\nu\left(r, D_{l,[S]}^{n}(f * g)\right)}= \\
=\left(\frac{l_{1} l_{\nu\left(r, D_{l,[S]}^{n}(f * g)\right)-1}}{l_{\nu\left(r, D_{l,[S]}^{n}(f * g)\right)}}\right)^{n-m}\left(\frac{l_{1} l_{\nu\left(r, D_{l,[S]}^{m}(f * g)\right)-1}}{l_{\nu\left(r, D_{l,[S]}^{n}(f * g)\right)}}\right)^{n}\left|f_{\nu\left(r, D_{l,[S]}^{n}(f * g)\right.}\right|\left|g_{\nu\left(r, D_{l,[S]}^{n}(f * g)\right)}\right| r^{\nu\left(r, D_{l,[S]}^{n}(f * g)\right)} \leq \\
\leq\left(\frac{l_{1} l_{\nu\left(r, D_{l,[S]}^{n}(f * g)\right)-1}}{l_{\nu\left(r, D_{l,[S]}^{n}(f * g)\right)}}\right)^{n-m} \mu\left(r, D_{l,[S]}^{m}(f * g)\right)
\end{gathered}
$$

and

$$
\mu\left(r, D_{l,[S]}^{m}(f * g)\right) \leq\left(\frac{l_{1} l_{\nu\left(r, D_{l,[S]}^{m}(f * g)\right)-1}}{l_{\nu\left(r, D_{l,[S]}^{m}(f * g)\right)}}\right)^{m-n} \mu\left(r, D_{l,[S]}^{n}(f * g)\right) .
$$

Therefore, for $m>n$

$$
\left(\frac{l_{\nu\left(r, D_{l,[S]}^{m}(f * g)\right)}}{l_{1} l_{\nu\left(r, D_{l,[S]}^{m}(f * g)\right)-1}^{m}}\right)^{m-n} \leq \frac{\mu\left(r, D_{l,[S]}^{n}(f * g)\right)}{\mu\left(r, D_{l,[S]}^{m}(f * g)\right)} \leq\left(\frac{l_{\nu\left(r, D_{l,[S]}^{n}(f * g)\right)}}{l_{1} l_{\nu\left(r, D_{l,[S]}^{n}(f * g)\right)-1}}\right)^{m-n}
$$

and

$$
\ln \ln \frac{l_{\nu\left(r, D_{l,[S]}^{m}(f * g)\right)}}{l_{1} l_{\nu\left(r, D_{l,[S]}^{m}(f * g)\right)-1}^{m}} \leq \ln \ln \frac{\mu\left(r, D_{l,[S]}^{n}(f * g)\right)}{\mu\left(r, D_{l,[S]}^{m}(f * g)\right)}-\ln (m-n) \leq \ln \ln \frac{l_{\nu\left(r, D_{l,[S]}^{n}(f * g)\right)}}{l_{1} l_{\nu\left(r, D_{l,[S]}^{n}(f * g)\right)-1}} .
$$

From condition (7) it follows that $q_{1} \ln k \leq \ln \ln \frac{l_{k}}{l_{1} l_{k-1}} \leq Q_{1} \ln k$ for every $0<q_{1}<q \leq Q<$ $Q_{1}<+\infty$ and all $k \geq k_{0}\left(q_{1}, Q_{1}\right)$. Therefore, for all $r \geq r_{0}$

$$
\begin{gathered}
q_{1}(1+o(1)) \ln \nu\left(r, D_{l,[S]}^{m}(f * g)\right) \leq \ln \ln \frac{\mu\left(r, D_{l,[S]}^{n}(f * g)\right)}{\mu\left(r, D_{l,[S]}^{m}(f * g)\right)} \leq \\
\leq Q_{1}(1+o(1)) \ln \nu\left(r, D_{l,[S]}^{n}(f * g)\right), \quad r \rightarrow+\infty .
\end{gathered}
$$

Since $\alpha_{1} \in L_{s i}$, we obtain

$$
\begin{gathered}
(1+o(1)) \alpha_{1}\left(\ln \nu\left(r, D_{l,[S]}^{m}(f * g)\right)\right) \leq \alpha_{1}\left(\ln \ln \frac{\mu\left(r, D_{l,[S]}^{n}(f * g)\right)}{\mu\left(r, D_{l,[S]}^{m}(f * g)\right)}\right) \leq \\
\leq(1+o(1)) \alpha_{1}\left(\ln \nu\left(r, D_{l,[S]}^{n}(f * g)\right)\right)
\end{gathered}
$$


as $r \rightarrow+\infty$. The condition $\frac{d \beta^{-1}(c \alpha(x))}{d \ln x}=O(1)$ as $x \rightarrow+\infty$ for each $c \in(0,+\infty)$ implies that $\alpha(x)=o(\beta(x))$ as $x \rightarrow+\infty$. Therefore, by Lemma 3 in view of the condition $\alpha(x)=\alpha_{1}(\ln x)$ we get

$$
\begin{aligned}
& \left.\left.\left.\varrho_{\alpha, \beta}\left[D_{l,[S]}^{m}(f * g)\right)\right]=\varrho_{\alpha, \beta}\left[\ln \mu, D_{l,[S]}^{m}(f * g)\right)\right]=\varrho_{\alpha, \beta}\left[\nu, D_{l,[S]}^{m}(f * g)\right)\right] \leq \\
& \leq \varlimsup_{r \rightarrow+\infty} \frac{1}{\beta(\ln r)} \alpha\left(\ln \frac{\mu\left(r, D_{l,[S]}^{n}(f * g)\right)}{\mu\left(r, D_{l,[S]}^{m}(f * g)\right)}\right) \leq \\
& \left.\left.\left.\leq \varrho_{\alpha, \beta}\left[\nu, D_{l,[S]}^{n}(f * g)\right)\right]=\varrho_{\alpha, \beta}\left[\ln \mu, D_{l,[S]}^{n}(f * g)\right)\right]=\varrho_{\alpha, \beta}\left[D_{l,[S]}^{n}(f * g)\right)\right]
\end{aligned}
$$

and

$$
\begin{gathered}
\left.\left.\left.\lambda_{\alpha, \beta}\left[D_{l,[S]}^{m}(f * g)\right)\right]=\lambda_{\alpha, \beta}\left[\ln \mu, D_{l,[S]}^{m}(f * g)\right)\right]=\lambda_{\alpha, \beta}\left[\nu, D_{l,[S]}^{m}(f * g)\right)\right] \leq \\
\leq \varlimsup_{r \rightarrow+\infty} \frac{1}{\beta(\ln r)} \alpha\left(\ln \frac{\mu\left(r, D_{l,[S]}^{n}(f * g)\right)}{\mu\left(r, D_{l,[S]}^{m}(f * g)\right)}\right) \leq \\
\left.\left.\left.\leq \lambda_{\alpha, \beta}\left[\nu, D_{l,[S]}^{n}(f * g)\right)\right]=\lambda_{\alpha, \beta}\left[\ln \mu, D_{l,[S]}^{n}(f * g)\right)\right]=\lambda_{\alpha, \beta}\left[D_{l,[S]}^{n}(f * g)\right)\right] .
\end{gathered}
$$

The assumptions Theorem 1 imply the conditions of Lemma 2. Therefore, if $\varliminf_{k \rightarrow \infty} \sqrt[k]{\left|f_{k} g_{k}\right|}>0$ then from (5) we get

$$
\begin{gathered}
\left.\varrho_{\alpha, \beta}\left[D_{l,[S]}^{n}(f * g)\right)\right]=\varlimsup_{k \rightarrow \infty} \alpha(k) / \beta\left(\frac{1}{k}\left(\ln \left(\frac{l_{k}}{l_{1} l_{k-1}}\right)^{n}+\ln \frac{1}{\left|f_{k}\right|\left|g_{k}\right|}\right)\right)= \\
=\varlimsup_{k \rightarrow \infty} \alpha(k) / \beta\left(\frac{n}{k} \ln \frac{l_{k}}{l_{k-1}}+O(1)\right)=\varlimsup_{k \rightarrow \infty} \alpha(k) / \beta\left(\frac{1}{k} \ln \frac{l_{k}}{l_{k-1}}\right),
\end{gathered}
$$

because $\beta \in L_{s i}$. Therefore, (12) implies (8).

If $\left|f_{k} / f_{k+1}\right| \nearrow 1,\left|g_{k} / g_{k+1}\right| \nearrow 1$ and $l_{k-1} l_{k+1} / l_{k}^{2} \nearrow+\infty$ as $k_{0} \leq k \rightarrow \infty$ then $\left|c_{k} / c_{k+1}\right| \nearrow$ $+\infty$ as $k_{0} \leq k \rightarrow \infty$, where $c_{k}=\left(\frac{l_{1} l_{k-1}}{l_{k}}\right)^{n} f_{k} g_{k}$. Therefore, as above by Lemma 2 we obtain

$$
\left.\lambda_{\alpha, \beta}\left[D_{l,[S]}^{n}(f * g)\right)\right]=\varliminf_{k \rightarrow \infty} \alpha(k) / \beta\left(\frac{1}{k} \ln \frac{l_{k}}{l_{k-1}}\right),
$$

and thus, (13) implies (9).

Using equality (4), we can obtain various corollaries from Theorem 1. For example, the following statement is true.

Corollary 1. Let $n \in \mathbb{Z}_{+}$and $j \in \mathbb{N}$. If the conditions of Theorem 1 hold then

$$
\varlimsup_{r \rightarrow+\infty} \frac{1}{\beta(\ln r)} \alpha\left(\ln \frac{\mu\left(r, D_{l,[S]}^{n} f * D_{l,[S]}^{n} g\right.}{\mu\left(r, D_{l,[S]}^{n+j} f * D_{l,[S]}^{n+j} g\right.}\right)=\varlimsup_{k \rightarrow \infty} \frac{\alpha(k)}{\beta\left(\frac{1}{k} \ln \frac{l_{k}}{l_{k-1}}\right)}
$$

and by conditions $\left|f_{k} / f_{k+1}\right| \nearrow 1,\left|g_{k} / g_{k+1}\right| \nearrow 1$ and $l_{k-1} l_{k+1} / l_{k}^{2} \nearrow+\infty$ as $k_{0} \leq k \rightarrow \infty$ a similar formula is true for $\underline{\text { lim. }}$.

Remark 1. Choosing $\alpha(x)=\ln ^{+} x$ and $\beta(x)=x^{+}$from the definitions of $\varrho_{\alpha, \beta}[f]$ and $\lambda_{\alpha, \beta}[f]$ we get the definitions of the order $\varrho[f]=\varlimsup_{r \rightarrow+\infty} \frac{\ln \ln M(r, f))}{\ln r}$ and the lower order $\lambda[f]=$ $\varliminf_{r \rightarrow+\infty} \frac{\ln \ln M(r, f))}{\ln r}$ for entire function (1). The functions $\alpha(x)=\ln ^{+} x$ and $\beta(x)=x^{+}$do not satisfy the hypotheses of Theorem 1. 
However, it is known (see for example $[15,16,17]$ ) that for entire function $(1) \varrho[f]=$ $\varlimsup_{r \rightarrow+\infty} \frac{\ln \nu(r, f))}{\ln r}=\varlimsup_{k \rightarrow \infty} \frac{k \ln k}{-\ln \left|f_{k}\right|}$, and if, moreover, $\left|f_{k}\right| /\left|f_{k+1}\right| \nearrow+\infty$ as $k_{0} \leq k \rightarrow \infty$ then $\lambda[f]=\varliminf_{r \rightarrow+\infty} \frac{\ln \nu(r, f))}{\ln r}=\varliminf_{k \rightarrow \infty} \frac{k \ln k}{-\ln \left|f_{k}\right|}$. Therefore, (11) yields

$$
q_{1} \varrho\left[D_{l,[S]}^{m}(f * g)\right] \leq \varlimsup_{r \rightarrow+\infty} \frac{1}{\ln r} \ln \ln \frac{\mu\left(r, D_{l,[S]}^{n}(f * g)\right)}{\mu\left(r, D_{l,[S]}^{m}(f * g)\right)} \leq Q_{1} \varrho\left[D_{l,[S]}^{n}(f * g)\right] .
$$

Moreover, as in the proof Theorem 1, we get $\left.\varrho\left[D_{l,[S]}^{n}(f * g)\right)\right]=\varlimsup_{k \rightarrow \infty} \frac{k \ln k}{\ln \left(l_{k} / l_{k-1}\right)}$. Using (11), similar results can be obtained for lower limits. Therefore, by virtue of arbitrariness of $q_{1}$ and $Q_{1}$ we come to the next statement.

Proposition 1. Let $m>n \geq 1, \varliminf_{k \rightarrow \infty} \sqrt[k]{\left|f_{k} g_{k}\right|}>0$ and (7) hold. Then

$$
q \varlimsup_{k \rightarrow \infty} \frac{k \ln k}{\ln \left(l_{k} / l_{k-1}\right)} \leq \varlimsup_{r \rightarrow+\infty} \frac{1}{\ln r} \ln \ln \frac{\mu\left(r, D_{l,[S]}^{n}(f * g)\right)}{\mu\left(r, D_{l,[S]}^{m}(f * g)\right)} \leq Q \varlimsup_{k \rightarrow \infty} \frac{k \ln k}{\ln \left(l_{k} / l_{k-1}\right)},
$$

and if, moreover, $\left|f_{k} / f_{k+1}\right| \nearrow 1,\left|g_{k} / g_{k+1}\right| \nearrow 1$ and $l_{k-1} l_{k+1} / l_{k}^{2} \nearrow+\infty$ as $k_{0} \leq k \rightarrow \infty$ then

$$
q \varliminf_{k \rightarrow \infty} \frac{k \ln k}{\ln \left(l_{k} / l_{k-1}\right)} \leq \varliminf_{r \rightarrow+\infty} \frac{1}{\ln r} \ln \ln \frac{\mu\left(r, D_{l,[S]}^{n}(f * g)\right)}{\mu\left(r, D_{l,[S]}^{m}(f * g)\right)} \leq Q \underline{\lim _{k \rightarrow \infty}} \frac{k \ln k}{\ln \left(l_{k} / l_{k-1}\right)} .
$$

2.2. The case $\lim _{\mathbf{k} \rightarrow \infty} \sqrt[k]{l_{k} / l_{k-1}}=\mathbf{1}$. Unlike entire functions for functions (1) with $R[f]=1$ the maximal term can be bounded, and in order that $\mu(r, f) \uparrow+\infty$ as $r \uparrow 1$ it is necessary and sufficient that $\varlimsup_{k \rightarrow \infty}\left|f_{k}\right|=+\infty$. In the sequel, we will consider that $\varlimsup_{k \rightarrow \infty}\left|f_{k}\right|=+\infty$, $\varlimsup_{k \rightarrow \infty}\left|g_{k}\right|=+\infty,\left|f_{k}\right|>1$ and $\left|g_{k}\right|>1$ for all $k \geq k_{0}$.

For $\alpha \in L, \beta \in L$ and the function (1) with $R[f]=1$ the quantities

$$
\varrho_{\alpha, \beta}^{(1)}[f]:=\varrho_{\alpha, \beta}^{(1)}[\ln M, f]=\varlimsup_{r \uparrow 1} \frac{\alpha(\ln M(r, f))}{\beta(1 /(1-r))}, \quad \lambda_{\alpha, \beta}^{(1)}[f]:=\lambda_{\alpha, \beta}^{(1)}[\ln M, f]=\frac{\lim }{r \uparrow 1} \frac{\alpha(\ln M(r, f))}{\beta(1 /(1-r))}
$$

are called ([18]) the generalized order and the lower generalized order, respectively. If here we substitute $\ln \mu(r, f)$ or $\nu(r, f)$ instead of $\ln M(r, f)$ then we obtain the definitions of the quantities $\varrho_{\alpha, \beta}^{(1)}[\ln \mu, f], \lambda_{\alpha, \beta}^{(1)}[\ln \mu, f]$ and $\lambda_{\alpha, \beta}^{(1)}[\nu, f], \lambda_{\alpha, \beta}^{(1)}[\nu, f]$, respectively. The following lemma is true.

Lemma 4. Let $\alpha \in L_{s i}, \beta \in L_{s i}$ and for each $c \in(0,+\infty)$

$$
\varlimsup_{x \rightarrow+\infty} \frac{d \ln \beta^{-1}(c \alpha(x))}{d \ln x}<1, \quad \lim _{x \rightarrow+\infty} \frac{\alpha\left(x / \beta^{-1}(c \alpha(x))\right)}{\alpha(x)}=1 .
$$

Then

$$
\varrho_{\alpha, \beta}^{(1)}[f]=\varlimsup_{k \rightarrow \infty} \frac{\alpha(k)}{\beta\left(k / \ln \left|f_{k}\right|\right)} .
$$

If, moreover, $\left|f_{k} / f_{k+1}\right| \nearrow 1$ as $k_{0} \leq k \rightarrow \infty$ then

$$
\lambda_{\alpha, \beta}^{(1)}[f]=\varliminf_{k \rightarrow \infty} \frac{\alpha(k)}{\beta\left(k / \ln \left|f_{k}\right|\right)} .
$$


Formula (15) is proved in [18], and formula (16) follows from the corresponding formula for Dirichlet series with finite abscissa of absolute convergence proved in [19, 20].

The following lemma also is proved in [14].

Lemma 5. If $\alpha \in L_{s i}, \beta \in L_{s i}$ and $\alpha(x)=o(\beta(x))$ as $x \rightarrow+\infty$ then $\varrho_{\alpha, \beta}^{(1)}[f]=\varrho_{\alpha, \beta}^{(1)}[\ln \mu, f]$ and $\lambda_{\alpha, \beta}^{(1)}[f]=\lambda_{\alpha, \beta}^{(1)}[\ln \mu, f]$. If, moreover, $\alpha\left(e^{x}\right) \in L_{s i}$ then $\varrho_{\alpha, \beta}^{(1)}[\ln \mu, f]=\varrho_{\alpha, \beta}^{(1)}[\nu, f]$ and $\lambda_{\alpha, \beta}^{(1)}[\ln \mu, f]=\lambda_{\alpha, \beta}^{(1)}[\nu, f]$.

The following analog of Theorem 1 is hold.

Theorem 2. Let $m>n \geq 1, \alpha\left(e^{x}\right) \in L_{s i}, \beta \in L_{s i}$ and (12) hold. Suppose that

$$
l_{k-1} / l_{k} \asymp k, \quad k \rightarrow \infty .
$$

Then

$$
\varlimsup_{r \uparrow 1} \frac{1}{\beta(1 /(1-r))} \alpha\left(\sqrt[m-n]{\frac{\mu\left(r, D_{l,[S]}^{m}(f * g)\right)}{\mu\left(r, D_{l,[S]}^{n}(f * g)\right)}}\right)=\max \left\{\varrho_{\alpha, \beta}^{(1)}[f], \varrho_{\alpha, \beta}^{(1)}[g]\right\}
$$

and if, moreover, $\left|f_{k} / f_{k+1}\right| \nearrow 1,\left|g_{k} / g_{k+1}\right| \nearrow 1$ and $l_{k-1} l_{k+1} / l_{k}^{2} \nearrow 1$ as $k_{0} \leq k \rightarrow \infty$ then

$$
\begin{gathered}
\max \left\{\lambda_{\alpha, \beta}^{(1)}[f], \lambda_{\alpha, \beta}^{(1)}[g]\right\} \leq \varlimsup_{r \uparrow 1} \frac{1}{\beta(1 /(1-r))} \alpha\left(\sqrt[m-n]{\frac{\mu\left(r, D_{l,[S]}^{m}(f * g)\right)}{\mu\left(r, D_{l,[S]}^{n}(f * g)\right)}}\right) \leq \\
\leq \min \left\{\max \left\{\lambda_{\alpha, \beta}^{(1)}[f], \varrho_{\alpha, \beta}^{(1)}[g]\right\}, \max \left\{\lambda_{\alpha, \beta}^{(1)}[g], \varrho_{\alpha, \beta}^{(1)}[f]\right\}\right\} .
\end{gathered}
$$

Proof. From (10) for $m>n$ we get

$$
\frac{l_{1} l_{\nu\left(r, D_{l,[S]}^{n}(f * g)\right)-1}}{l_{\nu\left(r, D_{l,[S]}^{n}(f * g)\right)}} \leq \sqrt[m-n]{\frac{\mu\left(r, D_{l,[S]}^{m}(f * g)\right)}{\mu\left(r, D_{l,[S]}^{n}(f * g)\right)}} \leq \frac{l_{1} l_{\nu\left(r, D_{l,[S]}^{m}(f * g)\right)-1}}{l_{\nu\left(r, D_{l,[S]}^{m}(f * g)\right)}} .
$$

Since in view of (17) there exist $0<q \leq Q<+\infty$ such that $q k \leq l_{1} l_{k-1} / l_{k} \leq Q k$, hence we obtain

$$
q \nu\left(r, D_{l,[S]}^{n}(f * g)\right) \leq \sqrt[m-n]{\frac{\mu\left(r, D_{l,[S]}^{m}(f * g)\right)}{\mu\left(r, D_{l,[S]}^{n}(f * g)\right)}} \leq Q \nu\left(r, D_{l,[S]}^{m}(f * g)\right) .
$$

We remark that since $\varlimsup_{x \rightarrow+\infty} \frac{d \ln y \beta^{-1}(c \alpha(x))}{d \ln x}<1$ as $x \rightarrow+\infty$ for each $c \in(0,+\infty)$, we have $\alpha(x)=o\left(\beta(x)\right.$ and $\frac{\beta^{-1}(c \alpha(x)) \ln x}{x} \rightarrow 0$ as $x \rightarrow+\infty$ for each $c \in(0,+\infty)$. Therefore, in view of the condition $\alpha\left(e^{x}\right) \in L_{s i}$ by Lemma 5 we get

$$
\begin{gathered}
\left.\left.\left.\varrho_{\alpha, \beta}^{(1)}\left[D_{l,[S]}^{n}(f * g)\right)\right]=\varrho_{\alpha, \beta}^{(1)}\left[\ln \mu, D_{l,[S]}^{n}(f * g)\right)\right]=\varrho_{\alpha, \beta}^{(1)}\left[\nu, D_{l,[S]}^{n}(f * g)\right)\right] \leq \\
\leq \varlimsup_{r \uparrow 1} \frac{1}{\beta(1 /(1-r))} \alpha\left(\sqrt[m-n]{\frac{\mu\left(r, D_{l,[S]}^{m}(f * g)\right)}{\mu\left(r, D_{l,[S]}^{n}(f * g)\right)}}\right) \leq \\
\left.\left.\left.\leq \varrho_{\alpha, \beta}^{(1)}\left[\nu, D_{l,[S]}^{m}(f * g)\right)\right]=\varrho_{\alpha, \beta}^{(1)}\left[\ln \mu, D_{l,[S]}^{m}(f * g)\right)\right]=\varrho_{\alpha, \beta}^{(1)}\left[D_{l,[S]}^{m}(f * g)\right)\right]
\end{gathered}
$$

and

$$
\left.\left.\left.\lambda_{\alpha, \beta}^{(1)}\left[D_{l,[S]}^{n}(f * g)\right)\right]=\lambda_{\alpha, \beta}^{(1)}\left[\ln \mu, D_{l,[S]}^{n}(f * g)\right)\right]=\lambda_{\alpha, \beta}^{(1)}\left[\nu, D_{l,[S]}^{n}(f * g)\right)\right] \leq
$$




$$
\begin{gathered}
\leq \frac{\lim }{r \uparrow 1} \frac{1}{\beta(1 /(1-r))} \alpha\left(\sqrt[m-n]{\frac{\mu\left(r, D_{l,[S]}^{m}(f * g)\right)}{\mu\left(r, D_{l,[S]}^{n}(f * g)\right)}}\right) \leq \\
\left.\left.\left.\leq \lambda_{\alpha, \beta}^{(1)}\left[\nu, D_{l,[S]}^{m}(f * g)\right)\right]=\lambda_{\alpha, \beta}^{(1)}\left[\ln \mu, D_{l,[S]}^{m}(f * g)\right)\right]=\lambda_{\alpha, \beta}^{(1)}\left[D_{l,[S]}^{m}(f * g)\right)\right] .
\end{gathered}
$$

Since $l_{1} l_{k-1} / l_{k} \geq q k \geq 1,\left|f_{k}\right|>1$ and $\left|g_{k}\right|>1$ for all $k \geq k_{0}$, we have $\left(\frac{l_{1} l_{k-1}}{l_{k}}\right)^{n}\left|f_{k}\right|\left|g_{k}\right| \geq$ $\left|f_{k}\right|$ and, similarly, $\left(\frac{l_{1} l_{k-1}}{l_{k}}\right)^{n}\left|f_{k}\right|\left|g_{k}\right| \geq\left|g_{k}\right|$ for all $k \geq k_{0}$. Therefore, $\mu\left(r, D_{l,[S]}^{n}(f * g)\right) \geq$ $(1+o(1)) \mu(r, f)$ and $\mu\left(r, D_{l,[S]}^{n}(f * g)\right) \geq(1+o(1)) \mu(r, g)$ as $r \uparrow 1$. Therefore, by Lemma 5 we get

$$
\left.\left.\varrho_{\alpha, \beta}^{(1)}\left[D_{l,[S]}^{n}(f * g)\right)\right] \geq \max \left\{\varrho_{\alpha, \beta}^{(1)}[f], \varrho_{\alpha, \beta}^{(1)}[g]\right\}, \lambda_{\alpha, \beta}^{(1)}\left[D_{l,[S]}^{n}(f * g)\right)\right] \geq \max \left\{\lambda_{\alpha, \beta}^{(1)}[f], \lambda_{\alpha, \beta}^{(1)}[g]\right\} .
$$

On the other hand, if $\varrho_{\alpha, \beta}^{(1)}[f]<+\infty$ and $\varrho_{\alpha, \beta}^{(1)}[g]<+\infty$ then by Lemma $4 \ln \left|f_{k}\right| \leq$ $\frac{k}{\beta^{-1}\left(\alpha(k) / \varrho_{1}\right)}$ and $\ln \left|g_{k}\right| \leq \frac{k}{\beta^{-1}\left(\alpha(k) / \varrho_{2}\right)}$ for every $\varrho_{1} \in\left(\varrho_{\alpha, \beta}^{(1)}[f],+\infty\right), \varrho_{2} \in\left(\varrho_{\alpha, \beta}^{(1)}[g],+\infty\right)$ and all $k \geq k_{0}$. Therefore, since $\frac{\beta^{-1}(c \alpha(x)) \ln x}{x} \rightarrow 0$ as $x \rightarrow+\infty$ for each $c \in(0,+\infty)$, we obtain

$$
\begin{aligned}
n \ln \frac{l_{1} l_{k-1}}{l_{k}}+\ln \left|f_{k}\right| & +\ln \left|g_{k}\right| \leq n \ln \left(l_{1} Q k\right)+\frac{k}{\beta^{-1}\left(\alpha(k) / \varrho_{1}\right)}+\frac{k}{\beta^{-1}\left(\alpha(k) / \varrho_{2}\right)} \leq \\
& \leq \frac{(2+o(1)) k}{\beta^{-1}\left(\alpha(k) / \max \left\{\varrho_{1}, \varrho_{2}\right\}\right)}, \quad k \rightarrow \infty,
\end{aligned}
$$

whence it follows that $\left.\varrho_{\alpha, \beta}^{(1)}\left[D_{l,[S]}^{n}(f * g)\right)\right] \leq \max \left\{\varrho_{1}, \varrho_{2}\right\}$, i. e. in view of the arbitrariness of $\varrho_{1}$ and $\varrho_{2}$ we get

$$
\left.\varrho_{\alpha, \beta}^{(1)}\left[D_{l,[S]}^{n}(f * g)\right)\right] \leq \max \left\{\varrho_{\alpha, \beta}^{(1)}[f], \varrho_{\alpha, \beta}^{(1)}[g]\right\} .
$$

Inequalities (21), (23) and (24) yield (18).

If $\left|f_{k} / f_{k+1}\right| \nearrow 1,\left|g_{k} / g_{k+1}\right| \nearrow 1$ and $l_{k-1} l_{k+1} / l_{k}^{2} \nearrow 1$ as $k_{0} \leq k \rightarrow \infty$ then $\left|c_{k} / c_{k+1}\right| \nearrow$ $+\infty$ as $k_{0} \leq k \rightarrow \infty$, where $c_{k}=\left(\frac{l_{1} l_{k-1}}{l_{k}}\right)^{n} f_{k} g_{k}$. Since $\ln \left|f_{k_{j}}\right| \leq \frac{k_{j}}{\beta^{-1}\left(\alpha\left(k_{j}\right) / \lambda\right)}$ for every $\lambda \in$ $\left(\lambda_{\alpha, \beta}^{(1)}[f],+\infty\right)$ and some sequence $\left(k_{j}\right) \uparrow+\infty$, as above, we have by Lemma 4

$$
\left.\lambda_{\alpha, \beta}^{(1)}\left[D_{l,[S]}^{n}(f * g)\right)\right] \leq \lim _{j \rightarrow \infty} \frac{\alpha\left(k_{j}\right)}{\beta\left(k_{j} / \ln \left|f_{k_{j}} g_{k_{j}}\right|\right)} \leq \max \left\{\lambda, \varrho_{2}\right\},
$$

in view of the arbitrariness of $\lambda$ and $\varrho_{2}$ we get $\left.\lambda_{\alpha, \beta}^{(1)}\left[D_{l,[S]}^{n}(f * g)\right)\right] \leq \max \left\{\lambda_{\alpha, \beta}^{(1)}[f], \varrho_{\alpha, \beta}^{(1)}[g]\right\}$. Similarly, $\left.\lambda_{\alpha, \beta}^{(1)}\left[D_{l,[S]}^{n}(f * g)\right)\right] \leq \max \left\{\lambda_{\alpha, \beta}^{(1)}[g], \varrho_{\alpha, \beta}^{(1)}[f]\right\}$. Therefore,

$$
\left.\lambda_{\alpha, \beta}^{(1)}\left[D_{l,[S]}^{n}(f * g)\right)\right] \leq \min \left\{\max \left\{\lambda_{\alpha, \beta}^{(1)}[f], \varrho_{\alpha, \beta}^{(1)}[g]\right\}, \max \left\{\lambda_{\alpha, \beta}^{(1)}[g], \varrho_{\alpha, \beta}^{(1)}[f]\right\}\right\} .
$$

Inequalities (22), (23) and (25) yield (19).

Remark 2. Choosing $\alpha(x)=\beta(x)=\ln ^{+} x$ from the definitions of $\varrho_{\alpha, \beta}^{(1)}[f]$ and $\lambda_{\alpha, \beta}^{(1)}[f]$ we get the definitions of the order

$$
\varrho^{(1)}[f]=\varlimsup_{r \uparrow 1} \frac{\left.\ln ^{+} \ln M(r, f)\right)}{\ln (1 /(1-r))}
$$

and the lower order

$$
\lambda^{(1)}[f]=\underline{\lim } \frac{\left.\ln ^{+} \ln M(r, f)\right)}{\ln (1 /(1-r))}
$$

for function (1) with $R[f]=1$. The functions $\alpha(x)=\beta(x)=\ln ^{+} x$ do not satisfy the assumptions of Theorem 2 . 
Now we have $([21])$

$$
\lambda^{(1)}[f] \leq \lambda^{(1)}[\nu, f] \leq \lambda^{(1)}[f]+1, \quad \varrho^{(1)}[f] \leq \varrho^{(1)}[\nu, f] \leq \varrho^{(1)}[f]+1 .
$$

We remark also that $([21,22])$

$$
\varrho^{(1)}[f]=\frac{\alpha^{*}[f]}{1-\alpha^{*}[f]}, \quad \alpha^{*}[f]:=\varlimsup_{k \rightarrow \infty} \frac{\ln ^{+} \ln \left|f_{k}\right|}{\ln k},
$$

and if $\left|f_{k}\right| /\left|f_{k+1}\right| \nearrow 1$ as $k_{0} \leq k \rightarrow \infty$ then

$$
\lambda^{(1)}[f]=\frac{\alpha_{*}[f]}{1-\alpha_{*}[f]}, \quad \alpha_{*}[f]:=\varliminf_{k \rightarrow \infty} \frac{\ln ^{+} \ln \left|f_{k}\right|}{\ln k} .
$$

From (20) we obtain

$$
\begin{gathered}
(m-n) \varrho^{(1)}\left[D_{l,[S]}^{n}(f * g)\right] \leq \varlimsup_{r \uparrow 1} \frac{1}{\ln (1 /(1-r))} \ln \frac{\mu\left(r, D_{l,[S]}^{m}(f * g)\right)}{\mu\left(r, D_{l,[S]}^{n}(f * g)\right)} \leq \\
\leq(m-n)\left(\varrho^{(1)}\left[D_{l,[S]}^{m}(f * g)\right]+1\right) .
\end{gathered}
$$

and

$$
\begin{gathered}
(m-n) \lambda^{(1)}\left[D_{l,[S]}^{n}(f * g)\right] \leq \varlimsup_{r \uparrow 1} \frac{1}{\ln (1 /(1-r))} \ln \frac{\mu\left(r, D_{l,[S]}^{m}(f * g)\right)}{\mu\left(r, D_{l,[S]}^{n}(f * g)\right)} \leq \\
\leq(m-n)\left(\lambda^{(1)}\left[D_{l,[S]}^{m}(f * g)\right]+1\right) .
\end{gathered}
$$

Since $l_{1} l_{k-1} / l_{k} \geq q k \geq 1,\left|f_{k}\right|>1$ and $\left|g_{k}\right|>1$ for all $k \geq k_{0}$, we have

$$
\begin{gathered}
\alpha^{*}\left[D_{l,[S]}^{n}(f * g)\right]=\varlimsup_{k \rightarrow \infty} \frac{\ln ^{+}\left(n \ln \left(l_{1} l_{k-1} / l_{k}\right)+\ln \left|f_{k}\right|+\ln \left|g_{k}\right|\right)}{\ln k} \geq \\
\geq \varlimsup_{k \rightarrow \infty} \frac{\ln ^{+} \min \left\{n q \ln k, \ln \left|f_{k}\right|\right\}}{\ln k}=\alpha^{*}[f],
\end{gathered}
$$

i. e. $\alpha^{*}\left[D_{l,[S]}^{n}(f * g)\right] \geq \max \left\{\alpha^{*}[f], \alpha^{*}[f]\right\}$ and, similarly, $\alpha_{*}\left[D_{l, S]}^{n}(f * g)\right] \geq \max \left\{\alpha_{*}[f], \alpha_{*}[g]\right\}$, whence

$$
\left.\varrho^{(1)}\left[D_{l,[S]}^{n}(f * g)\right] \geq \frac{\max \left\{\alpha^{*}[f], \alpha^{*}[g]\right\}}{1-\max \left\{\alpha^{*}[f], \alpha^{*}[g]\right\}}=\max \left\{\varrho^{(1)}[f], \varrho^{(1)}[g]\right)\right\}
$$

and similarly $\left.\lambda^{(1)}[f * g] \geq \max \left\{\lambda^{(1)}[f], \lambda^{(1)}[g]\right)\right\}$, provided $\left|f_{k} / f_{k+1}\right| \nearrow 1,\left|g_{k} / g_{k+1}\right| \nearrow 1$ and $l_{k-1} l_{k+1} / l_{k}^{2} \nearrow+\infty$ as $k_{0} \leq k \rightarrow \infty$.

On the other hand, $\ln \left|f_{k}\right| \leq k^{\alpha_{1}}$ and $\ln \left|g_{k}\right| \leq k^{\alpha_{2}}$ for every $\alpha_{1} \in\left(\alpha^{*}[f], 1\right), \alpha_{2} \in\left(\alpha^{*}[g], 1\right)$ and all $k \geq k_{0}$. Therefore,

$$
\alpha^{*}\left[D_{l,[S]}^{n}(f * g)\right] \leq \varlimsup_{k \rightarrow \infty} \frac{\ln (n Q \ln k)+\ln ^{+}\left(k^{\alpha_{1}}+k^{\alpha_{2}}\right)}{\ln k} \leq \max \left\{\alpha_{1}, \alpha_{2}\right\},
$$

i. e. in view of the arbitrariness of $\alpha_{1}$ and $\alpha_{2}$ we get $\alpha^{*}\left[D_{l,[S]}^{n}(f * g)\right] \leq \max \left\{\alpha^{*}[f], \alpha^{*}[g]\right\}$. Similarly we obtain $\alpha_{*}\left[D_{l,[S]}^{n}(f * g)\right] \leq \max \left\{\alpha_{*}[f], \alpha_{*}[g]\right\}$, whence as above we get

$$
\begin{gathered}
\varrho^{(1)}\left[D_{l,[S]}^{n}(f * g)\right] \leq \max \left\{\varrho^{(1)}[f], \varrho^{(1)}[g]\right\}, \\
\lambda^{(1)}\left[D_{l,[S]}^{n}(f * g)\right] \leq \min \left\{\max \left\{\lambda^{(1)}[f], \varrho^{(1)}[g]\right\}, \max \left\{\lambda^{(1)}[g], \varrho^{(1)}[f]\right\}\right\} .
\end{gathered}
$$

Therefore, in view of (26) and (27) we get the following statement. 
Proposition 2. Let $m>n \geq 1$ and (17) hold. Then

$$
\begin{gathered}
\left.(m-n) \max \left\{\varrho^{(1)}[f], \varrho^{(1)}[g]\right)\right\} \leq \varlimsup_{r \uparrow 1} \frac{1}{\ln (1 /(1-r))} \ln \frac{\mu\left(r, D_{l,[S]}^{m}(f * g)\right)}{\mu\left(r, D_{l,[S]}^{n}(f * g)\right)} \leq \\
\left.\leq(m-n)\left(\max \left\{\varrho^{(1)}[f], \varrho^{(1)}[g]\right)\right\}+1\right) .
\end{gathered}
$$

and if, moreover, $\left|f_{k} / f_{k+1}\right| \nearrow 1,\left|g_{k} / g_{k+1}\right| \nearrow 1$ and $l_{k-1} l_{k+1} / l_{k}^{2} \nearrow+\infty$ as $k_{0} \leq k \rightarrow \infty$ then

$$
\begin{gathered}
\left.(m-n) \max \left\{\lambda^{(1)}[f], \lambda^{(1)}[g]\right)\right\} \leq \frac{\lim }{r \uparrow 1} \frac{1}{\ln (1 /(1-r))} \ln \frac{\mu\left(r, D_{l,[S]}^{m}(f * g)\right)}{\mu\left(r, D_{l,[S]}^{n}(f * g)\right)} \leq \\
\leq(m-n)\left(\min \left\{\max \left\{\lambda^{(1)}[f], \varrho^{(1)}[g]\right\}, \max \left\{\lambda^{(1)}[g], \varrho^{(1)}[f]\right\}\right\}+1\right) .
\end{gathered}
$$

\section{Hadamard composition of two Gelfond-Leont'ev-Ruscheweyh derivatives.}

Let $f \in H, g \in H$ and $n \in \mathbb{N}$. Suppose that $\lim _{k \rightarrow \infty} \sqrt[k]{\frac{l_{k-1}}{l_{k}}}=q$. Then $\sqrt[k]{\frac{l_{k-1}}{l_{n+k-1}}} \rightarrow q^{n}$ as $k \rightarrow \infty$ and, as in the proof of Lemma 1, we get the following statement.

Lemma 6. If $f \in H, g \in H, n \in \mathbb{N}$ and there exists $\lim _{k \rightarrow \infty} \sqrt[k]{l_{k-1} / l_{k}}=q$ then $R\left[D_{l,[R]}^{n}(f * g)\right] \geq$ $q^{-n}$ and and if, moreover, $\lim _{k \rightarrow \infty} \sqrt[k]{\left|g_{k}\right|}=1$ then $R\left[D_{l,[R]}^{n}(f * g)\right]=q^{-n}$.

From Lemma 6 it follows that if $\sqrt[k]{l_{k} / l_{k-1}} \rightarrow \infty$ as $k \rightarrow \infty$ then $D_{l,[R]}^{n}(f * g) \in E$, and if $\sqrt[k]{l_{k} / l_{k-1}} \rightarrow 1$ as $k \rightarrow \infty$ then $D_{l,[R]}^{n}(f * g) \in H$. As in Section 1 , we consider only these two cases.

3.1. The case $\lim _{\mathbf{k} \rightarrow \infty} \sqrt[k]{l_{k} / l_{k-1}}=+\infty$. Since equation (4) is not satisfied for the Hadamard composition of Gelfond-Leont'ev-Ruscheweyhen derivatives, more variants arise in the study of the properties of these compositions. Let us start with an analogue of Theorem 1.

Theorem 3. Let the functions $\alpha$ and $\beta$ satisfy the assumptions of Theorem 1. Suppose that $\varliminf_{k \rightarrow \infty} \sqrt[k]{\left|f_{k} g_{k}\right|}>0$ and (7) holds. Then for $m>n \geq 1$

$$
\varlimsup_{r \rightarrow+\infty} \frac{1}{\beta(\ln r)} \alpha\left(\ln \frac{\mu\left(r, D_{l,[R]}^{n}(f * g)\right)}{\mu\left(r, D_{l,[R]}^{m}(f * g)\right)}\right)=\varlimsup_{k \rightarrow \infty} \frac{\alpha(k)}{\beta\left(\frac{1}{k} \ln \frac{l_{k}}{l_{k-1}}\right)}
$$

and if, moreover, $\left|f_{k} / f_{k+1}\right| \nearrow 1,\left|g_{k} / g_{k+1}\right| \nearrow 1$ and $l_{k-1} l_{k+1} / l_{k}^{2} \nearrow+\infty$ as $k_{0} \leq k \rightarrow \infty$ then

$$
\varliminf_{r \rightarrow+\infty} \frac{1}{\beta(\ln r)} \alpha\left(\ln \frac{\mu\left(r, D_{l,[R]}^{n}(f * g)\right)}{\mu\left(r, D_{l,[R]}^{m}(f * g)\right)}\right)=\varliminf_{k \rightarrow \infty} \frac{\alpha(k)}{\beta\left(\frac{1}{k} \ln \frac{l_{k}}{l_{k-1}}\right)} .
$$

Proof. Using the definitions of the maximal term and the central index, we have

$$
\begin{gathered}
\mu\left(r, D_{l,[R]}^{n}(f * g)\right)=\frac{l_{n} l_{\nu\left(r, D_{l,[R]}^{n}(f * g)\right)-1}}{l_{\nu\left(r, D_{l,[R]}^{n}(f * g)\right)+n-1}}\left|f_{\nu\left(r, D_{l,[R]}^{n}(f * g)\right.}\right|\left|g_{\nu\left(r, D_{l,[R]}^{n}(f * g)\right)}\right| r^{\nu\left(r, D_{l,[R]}^{n}(f * g)\right)}= \\
=\frac{l_{n} l_{\nu\left(r, D_{l,[R]}^{n}(f * g)\right)+m-1}}{l_{m} l_{\nu\left(r, D_{l,[R]}^{n}(f * g)\right)+n-1}} \frac{l_{m} l_{\nu\left(r, D_{l,[R]}^{n}(f * g)\right)-1}}{l_{\nu\left(r, D_{l,[R]}^{n}(f * g)\right)+m-1}}\left|f_{\nu\left(r, D_{l,[S]}^{n}(f * g)\right.}\right|\left|g_{\nu\left(r, D_{l,[S]}^{n}(f * g)\right)}\right| r^{\nu\left(r, D_{l,[S]}^{n}(f * g)\right)} \leq
\end{gathered}
$$




$$
\begin{gathered}
\leq \frac{l_{n} l_{\nu\left(r, D_{l,[R]}^{n}(f * g)\right)+m-1}}{l_{m} l_{\nu\left(r, D_{l,[R]}^{n}(f * g)\right)+n-1}} \mu\left(r, D_{l,[R]}^{m}(f * g)\right), \\
\mu\left(r, D_{l,[R]}^{m}(f * g)\right) \leq \frac{\left.l_{m} l_{\nu\left(r, D_{l,[R]}^{m}\right.}^{m}(f * g)\right)+n-1}{\left.l_{n} l_{\nu\left(r, D_{l,[R]}^{m}\right.}^{m}(f * g)\right)+m-1} \mu\left(r, D_{l,[R]}^{n}(f * g)\right) .
\end{gathered}
$$

Thus,

$$
\frac{l_{n} l_{\nu\left(r, D_{l,[R]}^{m}(f * g)\right)+m-1}}{l_{m} l_{\nu\left(r, D_{l,[R]}^{m}(f * g)\right)+n-1}^{m}} \leq \frac{\mu\left(r, D_{l,[R]}^{n}(f * g)\right)}{\mu\left(r, D_{l,[R]}^{m}(f * g)\right)} \leq \frac{l_{n} l_{\nu\left(r, D_{l,[R]}^{n}(f * g)\right)+m-1}}{l_{m} l_{\nu\left(r, D_{l,[R]}^{n}(f * g)\right)+n-1}} .
$$

In view of (7) for every $0<q_{1}<q \leq Q<Q_{1}<+\infty$ we have $k^{q_{1}} \leq \ln l_{k}-\ln l_{k-1} \leq k^{Q_{1}}$ for all $k \geq k_{0}\left(q_{1}, Q_{1}\right)$. Therefore,

$$
\begin{gathered}
\ln l_{\nu\left(r, D_{l,[R]}^{n}(f * g)\right)+m-1}-\ln l_{\nu\left(r, D_{l,[R]}^{n}(f * g)\right)+n-1}= \\
=\ln l_{\nu\left(r, D_{l,[R]}^{n}(f * g)\right)+m-1}-\ln l_{\nu\left(r, D_{l,[R]}^{n}(f * g)\right)+m-2}+\cdots+ \\
+\ln l_{\nu\left(r, D_{l,[R]}^{n}(f * g)\right)+m-(m-n)}-\ln l_{\nu\left(r, D_{l,[R]}^{n}(f * g)\right)+m-(m-n)-1} \leq \\
\leq\left(\nu\left(r, D_{l,[R]}^{n}(f * g)\right)+m-1\right)^{Q_{1}}+\cdots+\left(\nu\left(r, D_{l,[R]}^{n}(f * g)\right)+m-(m-n)\right)^{Q_{1}}= \\
=(m-n)(1+o(1)) \nu\left(r, D_{l,[R]}^{n}(f * g)\right)^{Q_{1}}, \quad r \rightarrow+\infty .
\end{gathered}
$$

Similarly, as $r \rightarrow+\infty$

$$
\ln l_{\nu\left(r, D_{l,[R]}^{n}(f * g)\right)+m-1}-\ln l_{\nu\left(r, D_{l,[R]}^{n}(f * g)\right)+n-1} \geq(m-n)(1+o(1)) \nu\left(r, D_{l,[R]}^{n}(f * g)\right)^{q_{1}} .
$$

Therefore, from (28) we obtain (11) with $D_{l,[R]}$ instead of $D_{l,[S]}$. Inequalities (11) imply (12) and (13) with $D_{l,[R]}$ instead of $D_{l,[S]}$. Finally, if $\varliminf_{k \rightarrow \infty} \sqrt[k]{\left|f_{k} g_{k}\right|}>0$ then by Lemma 2

$$
\left.\varrho_{\alpha, \beta}\left[D_{l,[R]}^{n}(f * g)\right)\right]=\varlimsup_{k \rightarrow \infty} \alpha(k) / \beta\left(\frac{1}{k} \ln \frac{l_{n+k-1}}{l_{1} l_{k-1}}+\ln \frac{1}{\left|f_{k}\right|\left|g_{k}\right|}\right)=\varlimsup_{k \rightarrow \infty} \alpha(k) / \beta\left(\frac{1}{k} \ln \frac{l_{k}}{l_{k-1}}\right)
$$

and, similarly,

$$
\left.\lambda_{\alpha, \beta}\left[D_{l,[R]}^{n}(f * g)\right)\right]=\varliminf_{k \rightarrow \infty} \alpha(k) / \beta\left(\frac{1}{k} \ln \frac{l_{k}}{l_{k-1}}\right) .
$$

Remark 3. Using the proof of Proposition 1 we get the following statement.

Proposition 3. Let $m>n \geq 1, \varliminf_{k \rightarrow \infty} \sqrt[k]{\left|f_{k} g_{k}\right|}>0$ and (7) hold. Then

$$
q \varlimsup_{k \rightarrow \infty} \frac{k \ln k}{\ln \left(l_{k} / l_{k-1}\right)} \leq \varlimsup_{r \rightarrow+\infty} \frac{1}{\ln r} \ln \ln \frac{\mu\left(r, D_{l,[R]}^{n}(f * g)\right)}{\mu\left(r, D_{l,[R]}^{m}(f * g)\right)} \leq Q \varlimsup_{k \rightarrow \infty} \frac{k \ln k}{\ln \left(l_{k} / l_{k-1}\right)},
$$

and if, moreover, $\left|f_{k} / f_{k+1}\right| \nearrow 1,\left|g_{k} / g_{k+1}\right| \nearrow 1$ and $l_{k-1} l_{k+1} / l_{k}^{2} \nearrow+\infty$ as $k_{0} \leq k \rightarrow \infty$ then

$$
q \underset{k \rightarrow \infty}{\varliminf_{k \rightarrow \infty}} \frac{k \ln k}{\ln \left(l_{k} / l_{k-1}\right)} \leq \varliminf_{r \rightarrow+\infty} \frac{1}{\ln r} \ln \ln \frac{\mu\left(r, D_{l,[R]}^{n}(f * g)\right)}{\mu\left(r, D_{l,[R]}^{m}(f * g)\right)} \leq Q \underset{\lim _{k \rightarrow \infty}}{\frac{k \ln k}{\ln \left(l_{k} / l_{k-1}\right)}},
$$


The following theorem is an analogue of Corollary 1.

Theorem 4. Let the functions $\alpha$ and $\beta$ satisfy the conditions of Theorem $1, n \in \mathbb{N}$ and $j \in \mathbb{N}$. Suppose that $\varliminf_{k \rightarrow \infty} \sqrt[k]{\left|f_{k} g_{k}\right|}>0$ and (7) holds. Then

$$
\varlimsup_{r \rightarrow+\infty} \frac{1}{\beta(\ln r)} \alpha\left(\ln \frac{\mu\left(r, D_{l,[R]}^{n} f * D_{l,[R]}^{n} g\right)}{\mu\left(r, D_{l,[R]}^{n+j} f * D_{l,[R]}^{n+j} g\right)}\right)=\varlimsup_{k \rightarrow \infty} \frac{\alpha(k)}{\beta\left(\frac{1}{k} \ln \frac{l_{k}}{l_{k-1}}\right)}
$$

and if, moreover, $\left|f_{k} / f_{k+1}\right| \nearrow 1,\left|g_{k} / g_{k+1}\right| \nearrow 1$ and $l_{k-1} l_{k+1} / l_{k}^{2} \nearrow+\infty$ as $k_{0} \leq k \rightarrow \infty$ then

$$
\varliminf_{r \rightarrow+\infty} \frac{1}{\beta(\ln r)} \alpha\left(\ln \frac{\mu\left(r, D_{l,[R]}^{n} f * D_{l,[R]}^{n} g\right)}{\mu\left(r, D_{l,[R]}^{n+j} f * D_{l,[R]}^{n+j} g\right)}\right)=\varliminf_{k \rightarrow \infty} \frac{\alpha(k)}{\beta\left(\frac{1}{k} \ln \frac{l_{k}}{l_{k-1}}\right)} .
$$

Proof. Since $\left(D_{l,[R]}^{n} f * D_{l,[R]}^{n} g\right)(z)=z+\sum_{k=2}^{\infty}\left(\frac{l_{k-1} l_{n}}{l_{n+k-1}}\right)^{2} f_{k} g_{k} z^{k}$, we have

$$
\begin{aligned}
& \mu\left(r, D_{l,[R]}^{n} f * D_{l,[R]}^{n} g\right)= \\
& =\left(\frac{l_{\nu\left(r, D_{l,[R]}^{n} f * D_{l,[R]}^{n} g\right)-1} l_{n}}{l_{n+\nu\left(r, D_{l,[R]}^{n} f * D_{l,[R]}^{n} g\right)-1}}\right)^{2}\left|f_{\nu\left(r, D_{l,[R]}^{n} f * D_{l,[R]}^{n} g\right)}\right|\left|g_{\nu\left(r, D_{l,[R]}^{n} f * D_{l,[R]}^{n} g\right)}\right| r^{\nu\left(r, D_{l,[R]}^{n} f * D_{l,[R]}^{n} g\right)}= \\
& =\left(\frac{l_{n} l_{\nu\left(r, D_{l,[R]}^{n} f * D_{l,[R]}^{n} g\right)+n+j-1}}{l_{n+j} l_{\nu\left(r, D_{l,[R]}^{n} f * D_{l,[R]}^{n} g\right)+n-1}}\right)^{2}\left(\frac{l_{n+j} l_{\nu\left(r, D_{l,[R]}^{n} f * D_{l,[R]}^{n} g\right)-1}}{l_{\nu\left(r, D_{l,[R]}^{n} f * D_{l,[R]}^{n} g\right)+n+j-1}}\right)^{2} \times \\
& \times\left|f_{\nu\left(r, D_{l,[R]}^{n} f * D_{l,[R]}^{n} g\right)}\right|\left|g_{\nu\left(r, D_{l,[R]}^{n} f * D_{l,[R]}^{n} g\right)}\right| r^{\nu\left(r, D_{l,[R]}^{n} f * D_{l,[R]}^{n} g\right)} \leq \\
& \leq\left(\frac{l_{n} l_{\nu\left(r, D_{l,[R]}^{n} f * D_{l,[R]}^{n} g\right)+n+j-1}}{l_{n+j} l_{\nu\left(r, D_{l,[R]}^{n} f * D_{l,[R]}^{n} g\right)+n-1}}\right)^{2} \mu\left(r, D_{l,[R]}^{n+j} f * D_{l,[R]}^{n+j} g\right)
\end{aligned}
$$

and, similarly,

$$
\mu\left(r, D_{l,[R]}^{n+j} f * D_{l,[R]}^{n+j} g\right) \leq\left(\frac{l_{n+j} l_{\nu\left(r, D_{l,[R]}^{n+j} f * D_{l,[R]}^{n+j} g\right)+n-1}}{l_{n} l_{\nu\left(r, D_{l,[R]}^{n+j} f * D_{l,[R]}^{n+j} g\right)+n+j-1}}\right)^{2} \mu\left(r, D_{l,[R]}^{n} f * D_{l,[R]}^{n} g\right) .
$$

Thus,

$$
\left(\frac{l_{n} l_{\nu\left(r, D_{l,[R]}^{n+j} f * D_{l,[R]}^{n+j} g\right)+n+j-1}}{l_{n+j} l_{\nu\left(r, D_{l,[R]}^{n+j} f * D_{l,[R]}^{n+j} g\right)+n-1}}\right)^{2} \leq \frac{\mu\left(r, D_{l,[R]}^{n} f * D_{l,[R]}^{n} g\right)}{\mu\left(r, D_{l,[R]}^{n+j} f * D_{l,[R]}^{n+j} g\right)} \leq\left(\frac{l_{n} l_{\nu\left(r, D_{l,[R]}^{n} f * D_{l,[R]}^{n} g\right)+n+j-1}}{l_{n+j} l_{\nu\left(r, D_{l,[R]}^{n} f * D_{l,[R]}^{n} g\right)+n-1}}\right)^{2} .
$$

Since in view of $(7) k^{q_{1}} \leq \ln l_{k}-\ln l_{k-1} \leq k^{Q_{1}}$ for every $0<q_{1}<q \leq Q<Q_{1}<+\infty$ and all $k \geq k_{0}\left(q_{1}, Q_{1}\right)$, as in the proof of Theorem 3 , we obtain

$$
\begin{gathered}
(1+o(1)) j \nu\left(r, D_{l,[R]}^{n} f * D_{l,[R]}^{n} g\right)^{q_{1}} \leq \ln l_{\nu\left(r, D_{l,[R]}^{n} f * D_{l,[R]}^{n} g\right)+n+j-1}-\ln l_{\nu\left(r, D_{l,[R]}^{n} f * D_{l,[R]}^{n} g\right)+n-1} \leq \\
\leq(1+o(1)) j \nu\left(r, D_{l,[R]}^{n} f * D_{l,[R]}^{n} g\right)^{Q_{1}}, \quad r \rightarrow+\infty .
\end{gathered}
$$

Therefore, (29) yields

$$
\left.(1+o(1)) q_{1} \ln \nu\left(r, D_{l,[R]}^{n+j} f * D_{l,[R]}^{n+j} g\right)\right) \leq \ln \ln \frac{\mu\left(r, D_{l,[R]}^{n} f * D_{l,[R]}^{n} g\right)}{\mu\left(r, D_{l,[R]}^{n+j} f * D_{l,[R]}^{n+j} g\right)} \leq
$$




$$
\leq(1+o(1)) Q_{1} \ln \nu\left(r, D_{l,[R]}^{n} f * D_{l,[R]}^{n} g\right), \quad r \rightarrow+\infty,
$$

i. e. we obtain (11) with $D_{l,[R]}^{n} f * D_{l,[R]}^{n} g$ instead of $D_{l,[S]}^{n}(f * g)$. This inequalities imply (12) and (13) with $D_{l,[R]}^{n} f * D_{l,[R]}^{n} g$ instead of $D_{l,[S]}^{n}(f * g)$. Finally, if $\underset{k \rightarrow \infty}{\lim } \sqrt[k]{\left|f_{k} g_{k}\right|}>0$ then by Lemma 2

$$
\varrho_{\alpha, \beta}\left[D_{l,[R]}^{n} f * D_{l,[R]}^{n} g\right]=\varlimsup_{k \rightarrow \infty} \alpha(k) / \beta\left(\frac{2}{k} \ln \frac{l_{n+k-1}}{l_{1} l_{k-1}}+\ln \frac{1}{\left|f_{k}\right|\left|g_{k}\right|}\right)=\varlimsup_{k \rightarrow \infty} \alpha(k) / \beta\left(\frac{1}{k} \ln \frac{l_{k}}{l_{k-1}}\right),
$$

and, similarly,

$$
\lambda_{\alpha, \beta}\left[D_{l,[R]}^{n} f * D_{l,[R]}^{n} g\right]=\varliminf_{k \rightarrow \infty} \alpha(k) / \beta\left(\frac{1}{k} \ln \frac{l_{k}}{l_{k-1}}\right) .
$$

Remark 4. Using (30), as in the proof of Proposition 1 we get the following statement.

Proposition 4. Let $n \in \mathbb{N}, j \in \mathbb{N}, \varliminf_{k \rightarrow \infty} \sqrt[k]{\left|f_{k} g_{k}\right|}>0$ and (7) hold. Then

$$
q \varlimsup_{k \rightarrow \infty} \frac{k \ln k}{\ln \left(l_{k} / l_{k-1}\right)} \leq \varlimsup_{r \rightarrow+\infty} \frac{1}{\ln r} \ln \ln \frac{\mu\left(r, D_{l,[R]}^{n} f * D_{l,[R]}^{n} g\right)}{\mu\left(r, D_{l,[R]}^{n+j} f * D_{l,[R]}^{n+j} g\right)} \leq Q \varlimsup_{k \rightarrow \infty} \frac{k \ln k}{\ln \left(l_{k} / l_{k-1}\right)},
$$

and if, moreover, $\left|f_{k} / f_{k+1}\right| \nearrow 1,\left|g_{k} / g_{k+1}\right| \nearrow 1$ and $l_{k-1} l_{k+1} / l_{k}^{2} \nearrow+\infty$ as $k_{0} \leq k \rightarrow \infty$ then

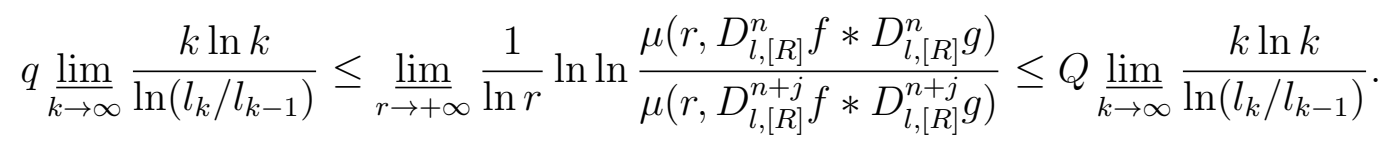

Theorem 5. Let the functions $\alpha$ and $\beta$ satisfy the conditions of Theorem $1, n \in \mathbb{N}$ and $j \in \mathbb{N}$. Suppose that $\varliminf_{k \rightarrow \infty} \sqrt[k]{\left|f_{k} g_{k}\right|}>0$ and (7) holds. Then

$$
\varlimsup_{r \rightarrow+\infty} \frac{1}{\beta(\ln r)} \alpha\left(\ln \frac{\mu\left(r, D_{l,[R]}^{n}(f * g)\right)}{\mu\left(r, D_{l,[R]}^{n+j} f * D_{l,[R]}^{n+j} g\right)}\right)=\varlimsup_{k \rightarrow \infty} \frac{\alpha(k)}{\beta\left(\frac{1}{k} \ln \frac{l_{k}}{l_{k-1}}\right)}
$$

and if, moreover, $\left|f_{k} / f_{k+1}\right| \nearrow 1,\left|g_{k} / g_{k+1}\right| \nearrow 1$ and $l_{k-1} l_{k+1} / l_{k}^{2} \nearrow+\infty$ as $k_{0} \leq k \rightarrow \infty$ then

$$
\varliminf_{r \rightarrow+\infty} \frac{1}{\beta(\ln r)} \alpha\left(\ln \frac{\mu\left(r, D_{l,[R]}^{n}(f * g)\right)}{\mu\left(r, D_{l,[R]}^{n+j} f * D_{l,[R]}^{n+j} g\right)}\right)=\varliminf_{k \rightarrow \infty} \frac{\alpha(k)}{\beta\left(\frac{1}{k} \ln \frac{l_{k}}{l_{k-1}}\right)} .
$$

Proof. As above, we have

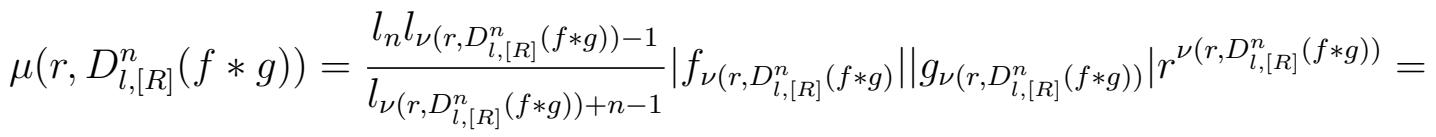

$$
\begin{aligned}
& \left.=\frac{l_{n} l_{\nu\left(r, D_{l,[R]}^{n}(f * g)\right)+n+j-1}^{2} l_{n+j}^{2} l_{\nu\left(r, D_{l,[R]}^{n}(f * g)\right)+n-1} l_{\nu\left(r, D_{l,[R]}^{n}(f * g)\right)-1}}{l_{\nu\left(r, D_{l,[R]}^{n}(f * g)\right)+n+j-1}}\right)^{2} \times \\
& \times\left|f_{\nu\left(r, D_{l,[R]}^{n}(f * g)\right.}\right|\left|g_{\nu\left(r, D_{l,[R]}^{n}(f * g)\right)}\right| r^{\nu\left(r, D_{l,[R]}^{n}(f * g)\right)} \leq
\end{aligned}
$$




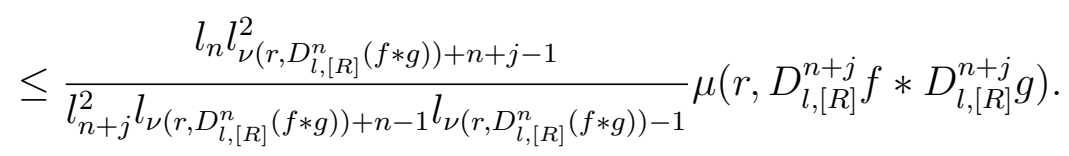

On the other hand,

$$
\begin{gathered}
\mu\left(r, D_{l,[R]}^{n+j} f * D_{l,[R]}^{n+j} g\right)=\left(\frac{l_{n+j} l_{\nu\left(r, D_{l,[R]}^{n+j} f * D_{l,[R]}^{n+j} g\right)-1}}{l_{\nu\left(r, \nu\left(r, D_{l,[R]}^{n+j} f * D_{l,[R]}^{n+j} g\right)+n+j-1\right.}}\right)^{2} \times \\
\times\left|f_{\nu\left(r, D_{l,[R]}^{n+j} f * D_{l,[R]}^{n+j} g\right)}\right|\left|g_{\nu\left(r, D_{l,[R]}^{n+j} f * D_{l,[R]}^{n+j} g\right)}\right| r^{\nu\left(r, D_{l,[R]}^{n+j} f * D_{l,[R]}^{n+j} g\right)}= \\
=\frac{l_{n+j}^{2+j} l_{\nu\left(r, D_{l,[R]}^{n+j} f * D_{l,[R]}^{n+j} g\right)-1} l_{\nu\left(r, D_{l,[R]}^{n+j} f * D_{l,[R]}^{n+j} g\right)+n-1}}{l_{n}^{2} l_{\nu\left(r, D_{l,[R]}^{n+j} f * D_{l,[R]}^{n+j} g\right)-1}} l_{\nu\left(r, D_{l,[R]}^{n+j} f * D_{l,[R]}^{n+j} g\right)+n-1} \times \\
\quad \times\left|f_{\nu\left(r, D_{l,[R]}^{n+j} f * D_{l,[R]}^{n+j} g\right)}\right|\left|g_{\nu\left(r, D_{l,[R]}^{n+j} f * D_{l,[R]}^{n+j} g\right)}\right| r^{\nu\left(r, D_{l,[R]}^{n+j} f * D_{l,[R]}^{n+j} g\right)} \leq \\
\leq \frac{\left.l_{n+j}^{2} l_{\nu\left(r, D_{l,[R]}^{n+j} f * D_{l,[R]}^{n+j} g\right)-1} l_{\nu\left(r, D_{l,[R]}^{n+j} f * D_{l,[R]}^{n+j} g\right)+n-1}^{n+j} g\right)+n\left(r, D_{l,[R]}^{n}(f * g)\right) .}{l_{n} l_{\nu\left(r, D_{l,[R]}^{n+j} f * D_{l,[R]}^{n+j} g\right)+n+j-1}^{2}} \mu
\end{gathered}
$$

Therefore,

$$
\begin{aligned}
& \frac{l_{n} l_{\nu\left(r, D_{l,[R]}^{n+j} f * D_{l,[R]}^{n+j} g\right)+n+j-1}^{2}}{l_{n+j}^{2} l_{\nu\left(r, D_{l,[R]}^{n+j} f * D_{l,[R]}^{n+j} g\right)-1} l_{\nu\left(r, D_{l,[R]}^{n+j} f * D_{l,[R]}^{n+j} g\right)+n-1}} \leq \\
& \leq \frac{\mu\left(r, D_{l,[R]}^{n}(f * g)\right)}{\mu\left(r, D_{l,[R]}^{n+j} f * D_{l,[R]}^{n+j} g\right)} \leq \frac{l_{n} l_{\nu\left(r, D_{l,[R]}^{n}(f * g)\right)+n+j-1}^{2} l_{n+j}^{2} l_{\nu\left(r, D_{l,[R]}^{n}(f * g)\right)+n-1} l_{\nu\left(r, D_{l,[R]}^{n}(f * g)\right)-1}}{l^{n}\left(D^{2}\right)}
\end{aligned}
$$

As in the proof of Theorem 3 we have

$$
\begin{aligned}
& \ln \frac{l_{\nu\left(r, D_{l,[R]}^{n}(f * g)\right)+n+j-1}^{2}}{l_{\nu\left(r, D_{l,[R]}^{n}(f * g)\right)+n-1} l_{\nu\left(r, D_{l,[R]}^{n}(f * g)\right)-1}}=\ln l_{\nu\left(r, D_{l,[R]}^{n}(f * g)\right)+n+j-1}-\ln l_{\nu\left(r, D_{l,[R]}^{n}(f * g)\right)+n-1}+ \\
& +\ln l_{\nu\left(r, D_{l,[R]}^{n}(f * g)\right)+n+j-1}-\ln l_{\nu\left(r, D_{l,[R]}^{n}(f * g)\right)-1} \leq(n+2 j)(1+o(1)) \nu\left(r, D_{l,[R]}^{n}(f * g)^{Q_{1}}\right.
\end{aligned}
$$

and, similarly,

$$
\ln \frac{l_{\nu\left(r, D_{l,[R]}^{n+j} f * D_{l,[R]}^{n+j} g\right)+n+j-1}^{2}}{l_{\nu\left(r, D_{l,[R]}^{n+j} f * D_{l,[R]}^{n+j} g\right)-1} l_{\nu\left(r, D_{l,[R]}^{n+j} f * D_{l,[R]}^{n+j} g\right)+n-1}} \geq(n+2 j)(1+o(1)) \nu\left(r, D_{l,[R]}^{n+j} f * D_{l,[R]}^{n+j} g\right)^{q_{1}}
$$

as $r \rightarrow+\infty$. Therefore, (31) implies

$$
\begin{gathered}
(1+o(1)) q_{1} \ln \nu\left(r, D_{l,[R]}^{n+j} f * D_{l,[R]}^{n+j} g\right) \leq \ln \ln \frac{\mu\left(r, D_{l,[R]}^{n}(f * g)\right)}{\mu\left(r, D_{l,[R]}^{n+j} f * D_{l,[R]}^{n+j} g\right)} \leq \\
\leq(1+o(1)) Q_{1} \ln \nu\left(r, D_{l,[R]}^{n}(f * g), \quad r \rightarrow+\infty .\right.
\end{gathered}
$$

The further proof of Theorem 5 is the same as the proof of Theorems 3 and 4 . 
Remark 5. Using (32), as above we get the following statement.

Proposition 5. Let $n \in \mathbb{N}, j \in \mathbb{N}$, $\varliminf_{k \rightarrow \infty} \sqrt[k]{\left|f_{k} g_{k}\right|}>0$ and (7) hold. Then

$$
q \varlimsup_{k \rightarrow \infty} \frac{k \ln k}{\ln \left(l_{k} / l_{k-1}\right)} \leq \varlimsup_{r \rightarrow+\infty} \frac{1}{\ln r} \ln \ln \frac{\mu\left(r, D_{l,[R]}^{n}(f * g)\right)}{\mu\left(r, D_{l,[R]}^{n+j} f * D_{l,[R]}^{n+j} g\right)} \leq Q \varlimsup_{k \rightarrow \infty} \frac{k \ln k}{\ln \left(l_{k} / l_{k-1}\right)},
$$

and if, moreover, $\left|f_{k} / f_{k+1}\right| \nearrow 1,\left|g_{k} / g_{k+1}\right| \nearrow 1$ and $l_{k-1} l_{k+1} / l_{k}^{2} \nearrow+\infty$ as $k_{0} \leq k \rightarrow \infty$ then

$$
q \varliminf_{k \rightarrow \infty} \frac{k \ln k}{\ln \left(l_{k} / l_{k-1}\right)} \leq \varliminf_{r \rightarrow+\infty} \frac{1}{\ln r} \ln \ln \frac{\mu\left(r, D_{l,[R]}^{n}(f * g)\right)}{\mu\left(r, D_{l,[R]}^{n+j} f * D_{l,[R]}^{n+j} g\right)} \leq Q \underset{k \rightarrow \infty}{\lim } \frac{k \ln k}{\ln \left(l_{k} / l_{k-1}\right)} .
$$

3.2. The case $\lim _{\mathbf{k} \rightarrow \infty} \sqrt[k]{l_{k} / l_{k-1}}=1$. As above, we will consider that

$$
\varlimsup_{k \rightarrow \infty}\left|f_{k}\right|=+\infty, \varlimsup_{k \rightarrow \infty}\left|g_{k}\right|=+\infty,\left|f_{k}\right|>1 \text { and }\left|g_{k}\right|>1 \text { for all } k \geq k_{0} \text {. }
$$

Theorem 6. Let $m>n \geq 1$ and the functions $\alpha$ and $\beta$ satisfy the conditions of Theorem 2 . Suppose that (17) holds. Then

$$
\varlimsup_{r \uparrow 1} \frac{1}{\beta(1 /(1-r))} \alpha\left(\sqrt[m-n]{\frac{\mu\left(r, D_{l,[R]}^{m}(f * g)\right)}{\mu\left(r, D_{l,[R]}^{n}(f * g)\right)}}\right)=\max \left\{\varrho_{\alpha, \beta}^{(1)}[f], \varrho_{\alpha, \beta}^{(1)}[g]\right\}
$$

and if, moreover, $\left|f_{k} / f_{k+1}\right| \nearrow 1,\left|g_{k} / g_{k+1}\right| \nearrow 1$ and $l_{k-1} l_{k+1} / l_{k}^{2} \nearrow 1$ as $k_{0} \leq k \rightarrow \infty$ then

$$
\begin{gathered}
\max \left\{\lambda_{\alpha, \beta}^{(1)}[f], \lambda_{\alpha, \beta}^{(1)}[g]\right\} \leq \varlimsup_{r \uparrow 1} \frac{1}{\beta(1 /(1-r))} \alpha\left(\sqrt[m-n]{\frac{\mu\left(r, D_{l,[R]}^{m}(f * g)\right)}{\mu\left(r, D_{l,[R]}^{n}(f * g)\right)}}\right) \leq \\
\leq \min \left\{\max \left\{\lambda_{\alpha, \beta}^{(1)}[f], \varrho_{\alpha, \beta}^{(1)}[g]\right\}, \max \left\{\lambda_{\alpha, \beta}^{(1)}[g], \varrho_{\alpha, \beta}^{(1)}[f]\right\}\right\} .
\end{gathered}
$$

Proof. In view of (17) there exist $0<q \leq Q<+\infty$ such that $q k \leq l_{k-1} / l_{k} \leq Q k$. From (28) we obtain

$$
\frac{l_{m} l_{\nu\left(r, D_{l,[R]}^{n}(f * g)\right)+n-1}}{l_{n} l_{\nu\left(r, D_{l,[R]}^{n}(f * g)\right)+m-1}} \leq \frac{\mu\left(r, D_{l,[R]}^{m}(f * g)\right)}{\mu\left(r, D_{l,[R]}^{n}(f * g)\right)} \leq \frac{l_{m} l_{\nu\left(r, D_{l,[R]}^{m}(f * g)\right)+n-1}}{l_{n} l_{\nu\left(r, D_{l,[R]}^{m}(f * g)\right)+m-1}},
$$

whence

$$
\begin{gathered}
(1+o(1)) \frac{l_{m}}{l_{n}}\left(q \nu\left(r, D_{l,[R]}^{n}(f * g)\right)\right)^{(m-n)} \leq \frac{\mu\left(r, D_{l,[R]}^{m}(f * g)\right)}{\mu\left(r, D_{l,[R]}^{n}(f * g)\right)} \leq \\
\leq(1+o(1)) \frac{l_{m}}{l_{n}}\left(Q \nu\left(r, D_{l,[R]}^{m}(f * g)\right)\right)^{(m-n)}, \quad r \uparrow 1, \\
q_{1} \nu\left(r, D_{l,[R]}^{n}(f * g)\right) \leq \sqrt[m-n]{\frac{\mu\left(r, D_{l,[R]}^{m}(f * g)\right)}{\mu\left(r, D_{l,[R]}^{n}(f * g)\right)}} \leq Q_{1} \nu\left(r, D_{l,[R]}^{m}(f * g)\right)
\end{gathered}
$$

for some $0<q_{1} \leq Q_{1}<+\infty$ and $r \in\left[r_{0}, 1\right)$, that is (20) holds with $q_{1}, Q_{1}$ instead of $q$, $Q$ and $D_{l,[R]}^{n}(f * g)$ instead of $D_{l,[S]}^{n}(f * g)$. Therefore, $(21)$ and $(22)$ hold with $D_{l,[R]}^{n}(f * g)$ instead of $D_{l,[S]}^{n}(f * g)$. 
Since $l_{k-1} / l_{k} \geq q k \geq 1,\left|f_{k}\right|>1$ and $\left|g_{k}\right|>1$ for all $k \geq k_{0}$, we have $\frac{l_{n} l_{k-1}}{l_{k+n-1}}\left|f_{k}\right|\left|g_{k}\right| \geq$ $l_{n}(q k)^{n}\left|f_{k}\right| \geq\left|f_{k}\right|$ and, similarly, $\frac{l_{n} l_{k-1}}{l_{k+n-1}}\left|f_{k}\right|\left|g_{k}\right| \geq\left|g_{k}\right|$ for all $k \geq k_{0}$. Therefore, as in the proof of Theorem 2 we get $(23)$ with $D_{l,[R]}^{n}(f * g)$ instead of $D_{l,[S]}^{n}(f * g)$.

On the other hand, for $\varrho_{1}>\varrho_{\alpha, \beta}^{(1)}[f]$ and $\varrho_{2}>\varrho_{\alpha, \beta}^{(1)}[g]$, as in the proof of Theorem 2 we have

$$
\begin{aligned}
\ln \frac{l_{n} l_{k-1}}{l_{k+n-1}}+\ln \left|f_{k}\right|+ & \ln \left|g_{k}\right| \leq \ln \left(l_{n}(Q k)^{n}\right)+\frac{k}{\beta^{-1}\left(\alpha(k) / \varrho_{1}\right)}+\frac{k}{\beta^{-1}\left(\alpha(k) / \varrho_{2}\right)} \leq \\
& \leq \frac{(2+o(1)) k}{\beta^{-1}\left(\alpha(k) / \max \left\{\varrho_{1}, \varrho_{2}\right\}\right)}, \quad k \rightarrow \infty,
\end{aligned}
$$

whence, as above we get $(24)$ and, similarly, (25) with $D_{l,[R]}^{n}(f * g)$ instead of $D_{l,[S]}^{n}(f * g)$. Thus, in (18) and (19) you can put $D_{l,[R]}^{n}(f * g)$ instead of $D_{l,[S]}^{n}(f * g)$.

Remark 6. For the usual orders the following proposition is true.

Proposition 6. Let $m>n \geq 1$ and (17) hold. Then

$$
\begin{gathered}
\left.(m-n) \max \left\{\varrho^{(1)}[f], \varrho^{(1)}[g]\right)\right\} \leq \varlimsup_{r \uparrow 1} \frac{1}{\ln (1 /(1-r))} \ln \frac{\mu\left(r, D_{l,[R]}^{m}(f * g)\right)}{\mu\left(r, D_{l,[R]}^{n}(f * g)\right)} \leq \\
\left.\leq(m-n)\left(\max \left\{\varrho^{(1)}[f], \varrho^{(1)}[g]\right)\right\}+1\right) .
\end{gathered}
$$

and if, moreover, $\left|f_{k} / f_{k+1}\right| \nearrow 1,\left|g_{k} / g_{k+1}\right| \nearrow 1$ and $l_{k-1} l_{k+1} / l_{k}^{2} \nearrow+\infty$ as $k_{0} \leq k \rightarrow \infty$ then

$$
\begin{gathered}
\left.(m-n) \max \left\{\lambda^{(1)}[f], \lambda^{(1)}[g]\right)\right\} \leq \frac{\lim }{r \uparrow 1} \frac{1}{\ln (1 /(1-r))} \ln \frac{\mu\left(r, D_{l,[R]}^{m}(f * g)\right)}{\mu\left(r, D_{l,[R]}^{n}(f * g)\right)} \leq \\
\leq(m-n)\left(\min \left\{\max \left\{\lambda^{(1)}[f], \varrho^{(1)}[g]\right\}, \max \left\{\lambda^{(1)}[g], \varrho^{(1)}[f]\right\}\right\}+1\right) .
\end{gathered}
$$

The proof of Proposition 6 is the same as that proof of Proposition 2. We just note that, since $\ln \left(l_{n} q k\right)^{n} \leq \frac{l_{n} l_{k-1}}{l_{k+n-1}} \leq \ln \left(l_{n} Q k\right)^{n}$, we get $\alpha^{*}\left[D_{l,[R]}^{n}(f * g)\right]=\max \left\{\alpha^{*}[f], \alpha^{*}[f]\right\}$ and $\alpha_{*}\left[D_{l,[R]}^{n}(f * g)\right]=\max \left\{\alpha_{*}[f], \alpha_{*}[g]\right\}$.

The following theorem holds for $\left.D_{l,[R]}^{n} f * D_{l,[R]}^{n} g\right)$.

Theorem 7. Let the functions $\alpha$ and $\beta$ satisfy the assumptions of Theorem $2, n \in \mathbb{N}$ and $j \in \mathbb{N}$. Suppose that (17) holds. Then

$$
\varlimsup_{r \uparrow 1} \frac{1}{\beta(1 /(1-r))} \alpha\left(\sqrt[2 j]{\frac{\mu\left(r, D_{l,[R]}^{n+j} f * D_{l,[R]}^{n+j} g\right)}{\mu\left(r, D_{l,[R]}^{n} f * D_{l,[R]}^{n} g\right)}}\right)=\max \left\{\varrho_{\alpha, \beta}^{(1)}[f], \varrho_{\alpha, \beta}^{(1)}[g]\right\}
$$

and if, moreover, $\left|f_{k} / f_{k+1}\right| \nearrow 1,\left|g_{k} / g_{k+1}\right| \nearrow 1$ and $l_{k-1} l_{k+1} / l_{k}^{2} \nearrow 1$ as $k_{0} \leq k \rightarrow \infty$ then

$$
\begin{gathered}
\max \left\{\lambda_{\alpha, \beta}^{(1)}[f], \lambda_{\alpha, \beta}^{(1)}[g]\right\} \leq \varlimsup_{r \uparrow 1} \frac{1}{\beta(1 /(1-r))} \alpha\left(\sqrt[2 j]{\frac{\mu\left(r, D_{l,[R]}^{n+j} f * D_{l,[R]}^{n+j} g\right)}{\mu\left(r, D_{l,[R]}^{n} f * D_{l,[R]}^{n} g\right)}}\right) \leq \\
\leq \min \left\{\max \left\{\lambda_{\alpha, \beta}^{(1)}[f], \varrho_{\alpha, \beta}^{(1)}[g]\right\}, \max \left\{\lambda_{\alpha, \beta}^{(1)}[g], \varrho_{\alpha, \beta}^{(1)}[f]\right\}\right\} .
\end{gathered}
$$


Proof. From (29) we get

$$
\left(\frac{l_{n+j} l_{\nu\left(r, D_{l,[R]}^{n} f * D_{l,[R]}^{n} g\right)+n-1}}{l_{n} l_{\nu\left(r, D_{l,[R]}^{n} f * D_{l,[R]}^{n} g\right)+n+j-1}}\right)^{2} \leq \frac{\mu\left(r, D_{l,[R]}^{n+j} f * D_{l,[R]}^{n+j} g\right)}{\mu\left(r, D_{l,[R]}^{n} f * D_{l,[R]}^{n} g\right)} \leq\left(\frac{l_{n+j} l_{\nu\left(r, D_{l,[R]}^{n+j} f * D_{l,[R]}^{n+j} g\right)+n-1}}{l_{n} l_{\nu\left(r, D_{l,[R]}^{n+j} f * D_{l,[R]}^{n+j} g\right)+n+j-1}}\right)^{2}
$$

whence in view of (17) as usual we get

$$
\begin{gathered}
(1+o(1)) \frac{l_{n+j}^{2}}{l_{n}^{2}}\left(q \nu\left(r, D_{l,[R]}^{n} f * D_{l,[R]}^{n} g\right)^{2 j} \leq \frac{\mu\left(r, D_{l,[R]}^{n+j} f * D_{l,[R]}^{n+j} g\right)}{\mu\left(r, D_{l,[R]}^{n} f * D_{l,[R]}^{n} g\right)} \leq\right. \\
\leq(1+o(1)) \frac{l_{n+j}^{2}}{l_{n}^{2}}\left(q \nu\left(r, D_{l,[R]}^{n+j} f * D_{l,[R]}^{n+j} g\right)^{2 j}, \quad r \uparrow 1,\right.
\end{gathered}
$$

i. e.

$$
q_{1} \nu\left(r, D_{l,[R]}^{n} f * D_{l,[R]}^{n} g\right) \leq \sqrt[2 j]{\frac{\mu\left(r, D_{l,[R]}^{n+j} f * D_{l,[R]}^{n+j} g\right)}{\mu\left(r, D_{l,[R]}^{n} f * D_{l,[R]}^{n} g\right)}} \leq Q_{1} \nu\left(r, D_{l,[R]}^{n+j} f * D_{l,[R]}^{n+j} g\right)
$$

for some $0<q_{1} \leq Q_{1}<+\infty$ and $r \in\left[r_{0}, 1\right)$. The further proof of Theorem 7 is the same as the proof of Theorem 6 .

We also note that by the usual method it is not difficult to prove the following statement.

Proposition 7. Let $n \in \mathbb{N}, j \in \mathbb{N}$ and (17) hold. Then

$$
\begin{aligned}
&\left.2 j \max \left\{\varrho^{(1)}[f], \varrho^{(1)}[g]\right)\right\} \leq \varlimsup_{r \uparrow 1} \frac{1}{\ln (1 /(1-r))} \ln \frac{\mu\left(r, D_{l,[R]}^{n+j} f * D_{l,[R]}^{n+j} g\right)}{\mu\left(r, D_{l,[R]}^{n} f * D_{l,[R]}^{n} g\right)} \leq \\
&\left.\leq 2 j\left(\max \left\{\varrho^{(1)}[f], \varrho^{(1)}[g]\right)\right\}+1\right) .
\end{aligned}
$$

and if, moreover, $\left|f_{k} / f_{k+1}\right| \nearrow 1,\left|g_{k} / g_{k+1}\right| \nearrow 1$ and $l_{k-1} l_{k+1} / l_{k}^{2} \nearrow 1$ as $k_{0} \leq k \rightarrow \infty$ then

$$
\begin{gathered}
\left.2 j \max \left\{\lambda^{(1)}[f], \lambda^{(1)}[g]\right)\right\} \leq \frac{\lim }{r \uparrow 1} \frac{1}{\ln (1 /(1-r))} \ln \frac{\mu\left(r, D_{l,[R]}^{n+j} f * D_{l,[R]}^{n+j} g\right)}{\mu\left(r, D_{l,[R]}^{n} f * D_{l,[R]}^{n} g\right)} \leq \\
\leq 2 j\left(\min \left\{\max \left\{\lambda^{(1)}[f], \varrho^{(1)}[g]\right\}, \max \left\{\lambda^{(1)}[g], \varrho^{(1)}[f]\right\}\right\}+1\right) .
\end{gathered}
$$

Finally, from (31) we obtain

$$
\begin{gathered}
\frac{l_{n+j}^{2} l_{\nu\left(r, D_{l,[R]}^{n}(f * g)\right)+n-1} l_{\nu\left(r, D_{l,[R]}^{n}(f * g)\right)-1} \leq \frac{\mu\left(r, D_{l,[R]}^{n+j} f * D_{l,[R]}^{n+j} g\right)}{\mu\left(r, D_{l,[R]}^{n}(f * g)\right)} \leq}{\left.l_{n} l_{\nu\left(r, D_{l,[R]}^{n}\right.}^{2}(f * g)\right)+n+j-1} \leq \\
\leq \frac{l_{n+j}^{2} l_{\nu\left(r, D_{l,[R]}^{n+j} f * D_{l,[R]}^{n+j} g\right)-1} l_{\nu\left(r, D_{l,[R]}^{n+j} f * D_{l,[R]}^{n+j} g\right)+n-1}}{\left.l_{n} l_{\nu\left(r, D_{l,[R]}^{2}\right.}^{2} f * D_{l,[R]}^{n+j} g\right)+n+j-1},
\end{gathered}
$$

whence in view of $(17)$

$$
(1+o(1)) \frac{l_{n+j}^{2}}{l_{n}}\left(q \nu\left(r, D_{l,[R]}^{n}(f * g)\right)\right)^{n+2 j} \leq \frac{\mu\left(r, D_{l,[R]}^{n+j} f * D_{l,[R]}^{n+j} g\right)}{\mu\left(r, D_{l,[R]}^{n}(f * g)\right)} \leq
$$




$$
\leq \frac{l_{n+j}^{2}}{l_{n}}\left(Q \nu\left(r, D_{l,[R]}^{n+j} f * D_{l,[R]}^{n+j} g\right)^{n+2 j}, \quad r \uparrow 1,\right.
$$

i. e.

$$
q_{1} \nu\left(r, D_{l,[R]}^{n}(f * g)\right) \leq \sqrt[n+2 j]{\frac{\mu\left(r, D_{l,[R]}^{n+j} f * D_{l,[R]}^{n+j} g\right)}{\mu\left(r, D_{l,[R]}^{n}(f * g)\right)}} \leq \leq Q_{1} \nu\left(r, D_{l,[R]}^{n+j} f * D_{l,[R]}^{n+j} g\right)
$$

for some $0<q_{1} \leq Q_{1}<+\infty$ and $r \in\left[r_{0}, 1\right)$.

Therefore, using the applied methodology above, we easily arrive at the correctness of the following two statements.

Theorem 8. Let the functions $\alpha$ and $\beta$ satisfy the assumptions of Theorem $2, n \in \mathbb{N}$ and $j \in \mathbb{N}$ and (17) hold. Then

$$
\varlimsup_{r \uparrow 1} \frac{1}{\beta(1 /(1-r))} \alpha\left(\sqrt[n+2 j]{\frac{\mu\left(r, D_{l,[R]}^{n+j} f * D_{l,[R]}^{n+j} g\right)}{\mu\left(r, D_{l,[R]}^{n}(f * g)\right)}}\right)=\max \left\{\varrho_{\alpha, \beta}^{(1)}[f], \varrho_{\alpha, \beta}^{(1)}[g]\right\}
$$

and if, moreover, $\left|f_{k} / f_{k+1}\right| \nearrow 1,\left|g_{k} / g_{k+1}\right| \nearrow 1$ and $l_{k-1} l_{k+1} / l_{k}^{2} \nearrow 1$ as $k_{0} \leq k \rightarrow \infty$ then

$$
\begin{gathered}
\max \left\{\lambda_{\alpha, \beta}^{(1)}[f], \lambda_{\alpha, \beta}^{(1)}[g]\right\} \leq \varlimsup_{r \uparrow 1} \frac{1}{\beta(1 /(1-r))} \alpha\left(\sqrt[n+2 j]{\frac{\mu\left(r, D_{l,[R]}^{n+j} f * D_{l,[R]}^{n+j} g\right)}{\mu\left(r, D_{l,[R]}^{n}(f * g)\right)}}\right) \leq \\
\leq \min \left\{\max \left\{\lambda_{\alpha, \beta}^{(1)}[f], \varrho_{\alpha, \beta}^{(1)}[g]\right\}, \max \left\{\lambda_{\alpha, \beta}^{(1)}[g], \varrho_{\alpha, \beta}^{(1)}[f]\right\}\right\} .
\end{gathered}
$$

Proposition 8. Let $n \in \mathbb{N}, j \in N$ and (17) hold. Then

$$
\begin{gathered}
\left.(n+2 j) \max \left\{\varrho^{(1)}[f], \varrho^{(1)}[g]\right)\right\} \leq \varlimsup_{r \uparrow 1} \frac{1}{\ln (1 /(1-r))} \ln \frac{\mu\left(r, D_{l,[R]}^{n+j} f * D_{l,[R]}^{n+j} g\right)}{\mu\left(r, D_{l,[R]}^{n}(f * g)\right)} \leq \\
\left.\leq(n+2 j)\left(\max \left\{\varrho^{(1)}[f], \varrho^{(1)}[g]\right)\right\}+1\right) .
\end{gathered}
$$

and if, moreover, $\left|f_{k} / f_{k+1}\right| \nearrow 1,\left|g_{k} / g_{k+1}\right| \nearrow 1$ and $l_{k-1} l_{k+1} / l_{k}^{2} \nearrow 1$ as $k_{0} \leq k \rightarrow \infty$ then

$$
\begin{gathered}
\left.(n+2 j) \max \left\{\lambda^{(1)}[f], \lambda^{(1)}[g]\right)\right\} \leq \frac{\lim }{r \uparrow 1} \frac{1}{\ln (1 /(1-r))} \ln \frac{\mu\left(r, D_{l,[R]}^{n+j} f * D_{l,[R]}^{n+j} g\right)}{\mu\left(r, D_{l,[R]}^{n}(f * g)\right)} \leq \\
\leq(n+2 j)\left(\min \left\{\max \left\{\lambda^{(1)}[f], \varrho^{(1)}[g]\right\}, \max \left\{\lambda^{(1)}[g], \varrho^{(1)}[f]\right\}\right\}+1\right) .
\end{gathered}
$$

\section{REFERENCES}

1. Gelfond A.O., Leont'ev A.F. On a generalisation of Fourier series// Matem. Sb. - 1951. - V.29, №3. P. 477-500. (in Russian)

2. Sălăgean G.St. Subclasses of univalent functions// Lecture Notes in Math. - 1983. - V.1013. - P. 362-372.

3. Ruscheweyh St. New criteria for univalent functions// Proc. Amer. Math. Soc. - 1975. - V.49. - P. 109115. 
4. Sheremeta M.M. On the maximal terms of successive Gelfond-Leont'ev-Sălăgean and Gelfond-Leont'evRuscheweyh derivatives of a function analytic in the unit disc// Mat. Stud. - 2012. - V.37, №1. - P. 58-64.

5. Hadamard J. Theoreme sur le series entieres// Acta Math. - 1899. - Bd.22. - S. 55-63.

6. Hadamard J. La serie de Taylor et son prolongement analitique // Scientia phys.-math. - 1901. - V.12. - P. 43-62.

7. Bieberbach L. Analytische Fortzetzung. - Berlin, 1955.

8. Korobeinik Yu.F., Mavrodi N.N. Singular points of the Hadamard composition// Ukr. Math. Zhourn. 1990. - V.42, №12. - P. 1711-1713. (in Russian)

9. Sen M.K. On some properties of an integral function $f * g / /$ Riv. Math. Univ. Parma (2). - 1967. - V.8. - P. 317-328.

10. Sen M.K. On the maximum term of a class of integral functions and its derivatives// Ann. Pol. Math. - 1970. - V.22. - P. 291-298.

11. Luhova L.L., Mulyava O.M., Sheremeta M.M. Properties of composition of Hadamard for GelfondLeont'ev derivatives of analytic functions// Ufa Mathematical Journal. - 2010. - V.2, №2. - P. 90-101. (in Russian)

12. Sheremeta M.N. Connection between the growth of the maximum of the modulus of an entire function and the moduli of the coefficients of its power series expansion// Izv. Vyssh. Uchebn. Zaved. Mat. - 1967. - №2 - P. 100-108. (in Russian)

13. Sheremeta M.M. Entire Dirichlet series. - K.: ISDO. - 1993. (in Ukrainian)

14. Sheremeta M.M., Mulyava O.M. Hadamard compositions of Gelfond-Leont'ev derivatives of analytic functions// Ukr. Math. Bull. - 2020. - V.17, №2. - P. 234-255.

15. Hadamard J. Essai sur l'étude des fonctions données par leur développement de Taylor// J. Math. Pures et Appl. - 1892. - V.12. - P. 154-186.

16. Valiron G. Integral functions. - Toulouse, 1923. - 354 p.

17. Whittaker J.M. The lower order of integral functions// J. London Math. Soc. - 1933. - V.8. - P. 10-17.

18. Sheremeta M.M. On the connection between the growth of a function analytic in a disk and the moduli of coefficients their Taylor series// DAN USSR - 1966. - №6. - P. 729-731. (in Russian)

19. Gal' Yu.M., Sheremeta M.M. On the growth of analytic fuctions in a half-plane given by Dirichlet series // DAN USSR, ser. A. - 1978. - №12. - P. 1964-1067. (in Russian)

20. Gal' Yu.M. On the growth of analytic fuctions given by Dirichlet series absolute convergent in a halfplane. - Drohobych. - 1980. - 40 pp. - Manuscript deposited at VINITI, № 4080-80 Dep.(in Russian)

21. Sons L.S. Regularity of growth and gap // J. Math. Anal. Appl. - 1968. - V.24. - P. 296-306.

22. Fujivara M. On the relation between $M(r)$ and the coefficients of a power series// Proc. Imp. Acad. Japan. - 1932. - V.8. - P. 220-223.

Ivan Franko National University of Lviv

Lviv, Ukraine

m.m.sheremeta@gmail.com 